Ann. Zootech., I966, (I), 89-II3.

MISE AU POINT BIBLIOGRAPHIQUE

\title{
ESTIMATION DE LA QUALITÉ DE LA GARCASSE DES AGNEAUX DE BOUCHERIE
}

\author{
J.-C. FLAMANT, R. BOCCARD \\ Station centrale de Génétique animale, \\ Laboratoire de Recherches sur la Viande, \\ Centre national de Recherches zootechniques, 78-Jouy-en-Josas
}

\section{PLAN}

I. - InTRODUCTION.

II. - Critères de qualité D’une carcasse.

I ${ }^{\circ}$ Poids de la carcasse.

$2^{\circ}$ Conformation de la carcasse

$3^{\circ}$ Composition de la carcasse.

$4^{\circ}$ Qualité de la viande

III. - Mesures des Caractéristiques d'Une carcasse.

${ }_{I} \mathbf{0}$ Méthodes directes
a) Poids de la carcasse
b) Conformation de la carcasse
c) Composition de la carcasse
d) Qualité de la viande

$2^{\circ}$ Méthodes indirectes

a) Mesure indirecte du poids de carcasse

b) Estimation de la conformation des carcasses sur l'animal vif

c) Mesures indirectes de la composition de la carcasse

- estimation par le rendement à l'abattage

- estimation par le poids de la carcasse

- estimation par la masse volumique de la carcasse

- estimation par la méthode au potassium-40

- estimation à l'aide de mesures linéaires diverses

- estimation par la composition des morceaux de la carcasse 


\section{IV. - Discussion \\ Io Relation poids-qualité des carcasses \\ $2^{0}$ Relation conformation-qualité des carcasses \\ $3^{0}$ Relation proportion des morceaux-qualité des carcasses

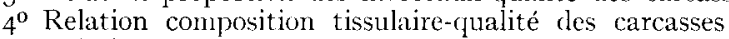 \\ $5^{\circ}$ Relation qualité de la viande-tualité des carcasses}

V. - CONCLUSION ẼT RÉSUMÁ.

\section{I. - INTRODUCTION}

La consommation française de viande d'origine ovine est caractérisée par une forte demande en viande jeune. Depuis quelques décennies la part relative des agneaux, dans l'effectif abattu chaque année, s'est fortement accrue. Cette tendance générale n'exclut pas la manifestation, d'une région à l'autre, de variations dans le type d'agneaux produits, en ce qui concerne l'âge et le poids. Ces variations résultent aussi bien des conditions agronomiques d'exploitation que des habitudes locales des centres de consommation.

Le problème de la qualité des carcasses fera l'objet de cette revue biblingraphique. Hammond a défini la qualité en la rapportant à la demande du consommateur exclusivement. Pour le producteur cette qualité se traduit par un prix : les carcasses de la qualité la plus recherchée obtiennent les meilleurs cours au kilogramme. A l'analyse la cualité d'une carcasse est le résultat complexe de divers critères exprimés couranment par les professionnels de la viande (bouchers et intermédiaires divers) et tenant compte de façon plus ou moins effective de gôtts supposés du consommateur : poids, conformation et composition de la carcasse (en tissus et en morceaux) et qualité de la viande.

Nous rappellerons tout d'abord les critères de jugement commerciaux. Puis nous ferons un inventaire des méthodes de découpe, et des mesures diverses sur les carcasses. Nous en viendrons à discuter l'influence réclle des critères invoqués sur la qualité des carcasses, et nous soulignerons les enseignements utiles au producteur d'agneaux de boucherie ì la lumière de ces conclusions. Nous dégagerons également quelques perspectives nouvelles de travaux de recherche.

\section{II. - CRITÈRES DE QUALITÉ D'UNE CARCASSE}

\section{$\mathrm{I}^{\circ}$ Poids de la carcasse}

Le poids des carcasses est sans doute l'un des éléments de la qualité les plus variables selon les régions. Certains marchés même, se caractérisent par des poids vifs situés dans une marge étroite, traduisant le désir d'obtenir des carcasses d'un poids moyen bien précis. Ainsi le marché de Nîmes est surtout un marché d'agneaux légers (poids à l'abattage $16-18 \mathrm{~kg}$ ) et les agneaux de lait du marché de Bordeaux pèsent de 8 à I $2 \mathrm{~kg}$. Par contre, dans le Nord on consomme de la viande d'agneaux d'un poids vif variant de 30 à $40 \mathrm{~kg}$. Ces rariations du poids optimum des carcasses pourraient être le résultat d'une demande variable pour la dimension optimum des morceaux résultant de la découpe. Les variations enregistrées en France se retrouvent sur le plan européen avec la même amplitude.

\section{$2^{\circ}$ Conformation de la carcasse}

Traditionnellement on recherche une carcasse aux reins larges et bien remplis, aux gigots épais et courts, plus larges que longs, et au cou peu développé en longueur : c'est-à-dire une carcasse large et courte. De plus, le boucher recherche des carcasses qui lui permettent de vendre la plus grande proportion possible de morceaux de première catégorie. Sa tendance à associer une bonne conformation à un plus fort pourcentage de gigot et de filet explique le rôle important de la conformation dans la valeur économique des carcasses. 


\section{$3^{\circ}$ Composition de la carcasse}

La composition de la carcasse devrait être le facteur prépondérant de la qualité puisque c'est elle qui conditionne en grande partie son prix de revient et sa valeur organoleptique. Mais la notion d'équilibre tissulaire " os-mucle-gras ", si souvent invoquée, est encore moins précise que la notion de conformation : en pratique son appréciation se traduit plutôt par des jugements portés sur le seul tissu adipeux.

a) Gras:

L'état d'engraissement est un caractère diversement apprécié selon les pays. Il joue un rôle important dans la détermination de la qualité et par conséquent du prix de la carcasse. Le boucher ne tolère pas une épaisseur de gras supérieure à la demande du consommateur qui l'obligerait à vendre l'excès à bas prix. La tradition professionnelle a établi une liaison entre l'importance du gras dans l'ensemble de la carcasse, et le développement du gras de rognon : les carcasses légèrement couvertes et avec un gras de rognon réduit sont les plus recherchées.

b) $O_{s}$ :

Les morceaux sont généralement vendus avec leur os (gigot, côtelette, collier....); aussi le consommateur demande-t-il des carcasses à ossature légère. Ce fait traduit la recherche du maximum de viande, recherche qui prend nettement plus d'importance quand il s'agit de juger le développement des tissus gras.

\section{$4^{\circ}$ Qualité de la viande}

Peu d'études ont été entreprises pour déterminer la qualité de la viande d'agneau. Dans la mesure où elle peut être appréciée, on connaît mal son incidence sur le jugement des carcasses.

En pratique la viande est soumise à deux impératifs de qualité :

a) la viande ne doit pas présenter un goût de suif prononcé (carcasses d'animaux âgés) ;

b) le gras doit être blanc. Les carcasses à gras jaune subissent une nette dépréciation.

Les races à fibres musculaires fines fournissent des carcasses généralement plus appréciées.

Trois caractéristiques de la carcasse semblent donc être déterminantes dans l'appréciation de sa qualité : son poids, sa conformation et son état d'engraissement.

Ia carcasse idéale, dans la limite des poids recherchés, se présente donc comme une carcasse large et courte, caractérisée par :

- la brièveté (et la légèreté) de ses rayons osseux, avec des masses musculaires bien développées et épaisses ;

- un gras de couverture sur les flancs, sur les gigots et sur le dos, qui ne dépasse pas une certaine épaisseur ( $\mathrm{x}$ à $3 \mathrm{~mm}$ en France) au delà de laquelle il dévalorise la carcasse.

\section{III. - CARACTÉRISTIQUES D'UNE CARCASSE}

L'étude systématique de la qualité des carcasses oblige à rechercher des méthodes d'appréciation hautement répétables et utilisables dans les travaux expérimentaux. Il importe de pouvoir comparer des carcasses sur des bases de jugement constantes dans le temps et dans l'espace. Le but recherché est donc de donner une expression quantitative des caractéristiques de carcasses. Trois qualités sont exigées des mesures :

$$
\begin{aligned}
& \mathrm{I}^{\mathrm{o}} \text { être de réalisation commode; } \\
& 2^{\mathrm{o}} \text { être hautement répétables; } \\
& 3^{\mathrm{o}} \text { être expressives. }
\end{aligned}
$$

S'il est nécessaire de retenir plusieurs mesures pour caractériser une carcasse, il est préférable de choisir autant que possible des mesures ayant des bases biologiques indépendantes. 


\section{I ${ }^{0}$ MÉTHODES DIRECTES}

\section{a) Poids de la carcasse}

La détermination du poids des carcasses s'effectue par pesée effectuée $24 \mathrm{~h}$ après l'abattage. Certains auteurs mesurent cependant le poids de carcasse "chaude " dont l'intérêt biologique est supérieur. Le poids de carcasse froide est plus habituellement utilisé dans les travaux (BRAY, 1963). Il est nécessaire alors de préciser exactement les conditions de conservation (humidité, température et durée).

\section{b) Conformation de la carcasse}

La conformation de la carcasse constitue un critère important de qualité pour les professionnels. HiRzel (I939) puis PALSSON (I939-I940), ont proposé de nombreuses mesures qui peuvent être effectuées sur une carcasse. Les mesures retenues peuvent servir à déterminer la forme de la carcasse : à l'aide de ces données on pourrait éventuellement dessiner la silhouette de la carcasse étudiée. Il s'en faut de beaucoup que toutes soient utilisables. Certaines sont de réalisation peu commode et de faible "répétabilité "(Barton, Phillips et Clarke, I949; Robinson, Binet et DoIG, I956). La variation de la plupart d'entre elles peut être expliquée par la variation du poids de la carcasse; elles représentent donc beaucoup plus le développement général de la carcasse (fortes corrélations avec le poids de carcasse : Boccard, Dumont et Peyron (I964), Twaites, Yeates et Pogue (1964). Mais le nombre de mesures nécessaires peut être réduit : il existe des relations constantes entre certaines dimensions d'une carcasse. Ainsi les résultats de Suck (I957), HundT (I959), Boccard, DumonT et Peyron (1964) montrent que les diverses mesures de largeur d'une carcasse sont en liaison étroite les unes avec les autres à poids de carcasse constant : une seule mesure suffit donc. Il en est de même pour la longueur. Par ailleurs HUNDT (I959) n'observe pas de relation entre la largeur du corps et les largeurs de poitrine et au bassin (sur agneaux vivants). TANEJA (1955) calcule une corrélation phénotypique hautement significative entre longueur et largeur du corps $(r=+0,43)$ sur des animaux Mérinos adultes. Plus récemment Boccard, Dumont et PEYron (I964) montrent à partir de mesures effectuées sur plus de 600 carcasses d'agneaux de toutes les races et de tout poids qu'une augmentation de la longueur du corps n'a pas d'influence, à poids de carcasse constant, sur la variation des largeurs du gigot et de celles du coffre. Cependant les corrélations calculées sont plus élevées entre longueur de carcasse et largeur du gigot qu'entre longueur de carcasse et largeur au coffre. On peut admettre en fonction de ces résultats qu'une mesure de longueur et une mesure de largeur sont nécessaires et suffisantes pour caractériser une carcasse.

Parmi les mesures retenues, les mesures " de conformation " se rapportent aux caractéristiques auxquelles les professionnels attachent le plus d'importance. Pour les carcasses d'agneaux l'appréciation porte essentiellement sur les gigots : la mesure F (longueur crosse-périnée) est la plus couramment retenue (Clarke et McMeekan, 1952 ; Coleman, I95I ; Haring et Schomburg, 1957; Boccard, Dumont et Peyron, 1958; Boccard et al. 1961; BocCard et Radowska, I963). La mesure P (longueur crosse-symphyse) peut être aussi utilisée (BARTon, PHILlips et Clarke, 1952). Boccard, Dumont et Peyron (I964) ont montré que la mesure $F$ variait peu en fonction du poids de la carcasse entre I I et $22 \mathrm{~kg}$ tandis que la largeur de la carcasse était en liaison étroite avec le poids. Il s'ensuit que pour des carcasses de même poids, la longueur F, sous l'influence d'autres facteurs que le poids de carcasse, peut exprimer le rebondi du gigot, conformément à l'opinion courante. Ce rebondi peut être encore représenté par le rapport $\mathrm{G} / \mathrm{F}\left(\frac{\text { largeur }}{\text { longueur }}\right)$, utilisé par CLARKE et McMeEKan (1952.)

Cependant les professionnels ne se contentent pas de définir les carcasses de bonne conformation par un gigot court. Ils désirent une carcasse large et courte. Aussi d'autres mesures « de conformation "sont-elles utilisées assez fréquemment et se rapportent à la longueur et à la largeur des carcasses (Barton, Phillips et Clarke, I959 ; Robinson, Binet et Doig, I956 ; Boccard, Dumont et Peyron, 1958). Boccard, Dumont et Peyron (1964) ont dressé un tableau des principales mesures utilisées expérimentalement. La méthode d'analyse factorielle (WRIGHT, I932 ; TANNER et BURT, I954; TAYLOR et RolliNs, 1963; Rouvier et VISSAC, I964) ou d'analyse en composantes principales (WRIGHT, I954; ROUVIER et RICARD, 1965), utilisées sur les carcasses d'agneaux devrait permettre de faire un choix objectif des mensurations pour exprimer la variabilité morphologique (développement général, variations de forme, par exemple).

ROBinson, BINET et DoIG (I956) dans une étude préliminaire, effectuent i6 mesures sur un premier lot de 50 carcasses d'agneaux australiens. Dix d'entre elles seulement sont retenues pour l'étude définitive portant sur 240 carcasses : F, longueur de gigots; Th, profondeur du thorax ; $\mathrm{BC}$, épaisseur du rein ; $\mathrm{P}$, épaisseur du flanc ; $\mathrm{K}$, longueur de carcasse ; Sh, épaisseur de l'épaule ; 
W, largeur aux épaules; E, largeur au coffre; $G$, largeur aux gigots; "Twist ", tour de jambe. Les mesures suivantes ont été éliminées par les auteurs parce que peu précises ou peu représentatives: longueur du quartier avant, longueur du filet, "remplissage " ou rebondi des gigots, épaisseur des gigots, tour de gigot, tour du rein.

Une certaine attention est aussi accordée à la forme de la section du muscle long dorsal (lon* gissimus dorsi), souvent considérée comme représentative du développement des masses musculaires. L'appréciation directe de la " noix de côtelette " nécessite une découpe de la carcasse. La section transversale s'effectue au niveau de la dernière côte, entre la I $_{2}$ et la ${ }^{\text {I }} 3^{\text {e }}$ (PALsson, I939; Bray, I963), ou entre la ${ }_{1}$ re et la $2^{\mathrm{e}}$ vertèbre lombaire (BoCCard, Dumont et l'EYron, 1958). On mesure A, longueur du grand axe de la section et B, longueur du petit axe perpendiculaire au premier. La surface peut être planimétrée sur calque ou sur photographie de la section. On effectue généralement la moyenne des mesures droite et gauche. On mesure aussi $\mathrm{C}$, épaisseur du gras dorsal au-dessus du longissimus dorsi (une seule mesure, ou la moyenne de 3 mesures en 3 points de la section). Ces mesures nécessitant une découpe sont évidemment de réalisation plus laborieuse que celles qui portent sur la carcasse entière. En outre les carcasses se trouvent dépréciées pour le commerce. Hammond (I936) a caractérisé la forme de la section du long dorsal par le shape index $(\mathrm{B} / \mathrm{A} \times \mathrm{ro0})$ qui constitue selon lui un indice de qualité. La représentativité de cet index sera discutée dans les chapitres suivants.

\section{c) Composition de la carcasse}

La connaissance de la composition des carcasses nécessite l'utilisation de mesures plus fines et plus délicates à réaliser que les mesures de conformation.

\section{Proportion des morceaux.}

Les découpes utilisées dans le commerce isolent un certain nombre de morceaux de valeurs différentes. Certaines carcasses présentent-elles un plus fort pourcentage de morceaux de première catégorie? Les bouchers répondent implicitement oui à cette question.

Des comparaisons valables ne sont permises entre carcasses, que si les différences dues à des imprécisions de découpe ont été éliminées. Aussi, avant d'entreprendre toute étude, un certain nombre d'auteurs ont-ils insisté sur la nécessité de définir une découpe de référence : l'isolement des divers morceaux doit se faire selon un processus normalisé et précis (PALsson, i939; Hankins, I947; Boccard et Dumont, 1955; Carroll et O'Carrol, 1964). Ces découpes s'inspirent le plus étroitement possible des découpes commerciales locales. La commercialisation des carcasses ainsi découpées rencontre moins de difficultés qu'avec une découpe qui ne correspondrait pas aux habitudes du marché, mais gêne parfois les comparaisons : les résultats obtenus dans un pays à l'aide d'une certaine découpe ne sont pas utilisables en toute rigueur par les chercheurs d'un autre pays utilisant une découpe de référence différente. En particulier, d'une école à l'autre, on appelle d'un même nom des morceaux totalement différents anatomiquement : ceci se manifeste surtout pour la découpe de l'épaule. Une entente internationale serait particulièrement utile dans ce domaine. (F.E.Z. I953).

La découpe parisienne a servi de modèle à la découpe utilisée en France par Boccard et DumonT (1955). Les morceaux suivants sont isolés :

- le "gigot entier " qui se divise lui-même en " selle » et "gigot raccourci ";

- le "filet " qui correspond à la région lombaire avec les parois abdominales adjacentes ;

- l'épaule, essentiellement composée du membre antérieur anatomique, ce qui ne correspond pas à la définition de l'épaule de la découpe anglo-saxonne;

- le "carré " (région des vertèbres dorsales et partie des côtes correspondantes) ;

- la poitrine et le " haut des côtelettes";

- le " collier".

Ces découpes sont effectuées sur $1 / 2$ carcasses en partant de l'hypothèse de la symétrie des carcasses des animaux jeunes.

\section{Composition en tissus.}

L'analyse directe des carcasses en tissus nécessite une dissection complète. On isole communément 3 groupes de tissus :

- le tissu gras;

- le tissu musculaire;

- les os auxquels quelquefois des auteurs ont joint les tendons, ligaments et cartilages, alors que les autres en ont fait un groupe spécial. 
La séparation de ces tissus dans les différentes régions du corps exige une très grande minutie et beaucoup de temps. Des spécialistes entraînés travaillent avec un pourcentage de pertes ne dépassant pas 2 p. Ioo.

\section{Composition chimique.}

La connaissance de la composition chimique d'une carcasse est souvent recherchée dans les études de nutrition. Mais elle peut aussi permettre une estimation de la composition de la carcasse en ses différents tissus.

Les diverses méthodes d'analyse utilisées diffèrent entre elles, essentiellement par l'origine, le nombre et la dimension des échantillons. L'une des méthodes les plus utilisées par les chercheurs anglo-saxons est la méthode dite "BARTON-KIRTON" (BARTON et KIRTON, I956-I958). Elle consiste à découper une demi-carcasse en fines lamelles de 3 à $6 \mathrm{~mm}$ d'épaisseur qui sont ensuite broyées ensemble. L'analyse porte ensuite sur des échantillons de $50 \mathrm{~g}$ de ce broyat. Cette méthode a inspiré la méthode officielle de l'A.O.A.C. (Association of Official Agricultural Chemists, I960) : celleci apparaît plus sensible pour l'estimation des cendres, les précisions obtenues pour les autres composants étant similaires. (KEMP et BARTON, 1965).

Munson et al. (1965) constituent, selon le même processus que Barton et Kirton, huit échantillons de $50 \mathrm{~g}$ chacun, mais à partir desquels ils constituent un seul échantillon de $5 \circ \mathrm{g}$ sur lequel porte l'analyse. Il en résulte une diminution de la part de variation due à l'échantillonnage, en particulier dans l'estimation de l'extrait à l'éther des carcasses. Mifford et Munson (1965), obtiennent des échantillons de $300 \mathrm{~g}$, à partir de $\mathrm{r} / 2$ carcasses, en constituant des émincés de plus en plus fins mélangés après chaque opération.

Kirton, BARTon et RAE (I962) montrent que la prise des échantillons sur une demi-carcasse seulement ne conduit pas à une évaluation biaisée de la composition chimique de la carcasse entière. Nous reviendrons sur cette question dans le paragraphe $2-c$, consacré à l'évaluation de la composition de la carcasse par la composition de morceaux individuels.

\section{d) Qualité de la viande}

La viande d'agneau étant une viande jeune, les problèmes tels que celui de la tendreté apparaissent accessoires face à ceux de la production, de la conformation ou de la composition des carcasses. Ne se pose pas non plus la question de la qualité technologique de la viande comme chez le porc. Les facteurs qui conditionnent la qualité de la viande, ct par suite de la carcasse d'agneau (saveur de la viande et couleur du gras) sont très facilement jugés sans qu'il soit besoin de faire appel à des tests élaborés. Il semble cependant qu'un nombre croissant de chercheurs s'attachent à étudier la qualité de la viande d'agneau selon les méthodes d'études employées pour les bovins. SCHEPER (I962) a fait un inventaire des divers travaux relatifs aux facteurs de qualité de la viande chez les différentes espèces. Dans la suite de cette revue bibliographique nous ne ferons qu'évoquer le problème de la qualité de viande, lorsqu'il interviendra effectivement sur la qualité des carcasses.

Les méthodes directes d'appréciation, décrites précédemment, permettent d'effectuer avec précision des mesures de conformation et de composition des carcasses.

Mais ces méthodes d'analyse sont telles qu'elles nécessitent l'abattage de l'animal, et jusqu'à la destruction partielle ou totale de la carcasse. Or, le sélectionneur a tout intérêt à obtenir des renseignements concernant la carcasse (conformation par exemple) à partir des données recueillies sur l'animal vivant. L'utilisation de méthodes indirectes d'appréciation de la carcasse doit permettre la conservation des géniteurs éventuellement intéressants ou, à défaut, le maintien de l'intégrité des carcasses.

D'autre part, on cherche toujours à apprécier la composition de la carcasse à partir de mesures effectuées sur l'extérieur de cette carcasse. L'estimation de la composition du corps par des caractéristiques mesurées sur l'animal vivant présente aussi un grand intérêt dans l'étude du développement.

\section{$2^{\circ}$ MÉTHODES INDIRECTES}

Deux catégories de mesures indirectes peuvent être distinguées :

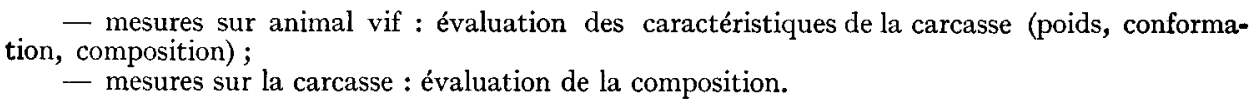




\section{a) Mesure indirecte du poids de carcasse}

Pour un animal déterminé le poids de la carcasse est lié au poids de l'animal vif. Mais cette liaison n'est pas suffisanment étroite pour permettre une bolme estimation du poids de carcasse par le poids vif mesuré peu avant l'abattage.

Le "rendement" (- Poids de la carcasse $\times 100)$ est variable d'un animal à l'autre. L'estimation du poids de carcasse par le poids à l'abattage est donc soumis à certaines imprécisions qui tiennent en particulier au remplissage viscéral des animaux au moment de l'abattage.

La barymétrie, autre système, a surtout pour but d'estimer le poids vif réel d'un animal à partir de certaines dimensions du corps. Delacik, POLY et Vissac (1955), rapportent les diverses mesures utilisées et les formules proposées sur les bovins. Il serait intéressant de pouvoir estimer de la même façon le poids de la carcasse à partir des mesures sur agneaux vifs. Mais dans la plupart des cas, la présence de la toison introduit une trop grande imprécision dans les mesures. KATADA et 'TAKEDA (1958) proposent 6 mesures d'estimation sur le vif, étroitement liées au poids vif et au poids de carcasse : profondeur de poitrine, largeur de poitrine, longueur du corps, longueur du rein, largeur aux hanches et largeur au trochanter.

\section{b) Estimation de la conformation des carcasses sur l'animal aif}

Le choix des mesures de conformation sur l'animal vif est orienté par le choix des mesures sur la carcasse. Aussi chiffre-t-on sur le vif, le plus souvent, les mesures homologues à celles qui caractérisent habituellement les carcasses. Ainsi le problème de représentativité des mesures est résolu par la détermination de relations entre mesures sur le vif et mesures sur la carcasse (KinaNDekar, McManus et Golistone, ig6.3). Cet asject semble intéresser plus spécialement la prédiction des caractéristiques de la section du muscle long dorsal et de l'épaisseur C du gras entre la $\mathbf{I}_{2} \mathbf{e}^{\mathrm{e}}$

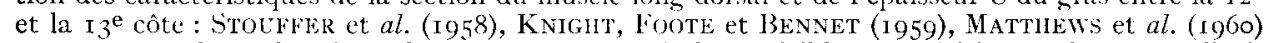
montrent que les estimations obtenues sont en général trop faibles en précision sauf peut-être l'utilisation des ultra sons (CaMpbell, STONAKFr et ESFlin, 1959; ZOIRINSKY et al., i96i ; I)AVIS et al., 1964) ce qui concorde avec les résultats obtenus sur d'autres espèces (voir tabl. I).

L'appréciation de la conformation sur animal vif est très liée aussi aux problèmes de l'amélioration génétiq̣ue de la production de viande : les béliers sélectionnés pour leur conformation vont-ils donner des agneaux dont les carcasses auront aussi une bonne conformation? Le choix des mensurations est donc aussi dépendant de leur héritabilité et dı mode de transmission de ces caractères (Hillman et al., 1962).

Par ailleurs une dizaine de mensurations (dont la hauteur au garrot) sont utilisées dans les études sur la croissance.

Il est nécessaire que les mesures, quelle que soit leur utilisation, soient de bonne précision. I)ans cette perspective, LARGE et 'TAYLER (I954) retiennent les trois mesures suivantes : longueur du métacarpe, largeur aux ilions, longueur du bassin. TANEJA (I955, a) sur 557 animaux calcule les coefficients de "répétabilité " suivants :

$\begin{array}{ll}\text { profondeur du corps } & 0,72 \\ \text { longueur du corps } & 0,61 \\ \text { largeur du corps } & 0,45 \\ \text { longueur du métacarpe } & 0,62\end{array}$

Il est bien évident que les mesures de faible précision ne peuvent servir d'index de prédiction, et que les héritabilités calculées à partir de telles données ne peuvent qu'être faibles, la variation aléatoire étant trop importante. Si on veut utiliser tout de même la mensuration, il conviendrait alors de considérer la movenne de plusieurs répétitions indépendantes de la mesure.

\section{c) Mesures indirectes de la composition de la carcasse}

Les mesures indirectes de la composition ont, compte tenu de l'intérêt de cette détermination, suscité le plus grand nombre de travaux, utilisant des méthodes fort variables et visant des objectifs très divers. Ces mesures, permettant d'estimer la composition en tissus sans mutiler la carcasse (aucune découpe), peuvent être utilisées en dehors du laboratoire si leur réalisation n'est pas trop complexe. Les méthodes nécessitant une découpe peuvent être de meilleure précision mais servent alors essentiellement d'étalon à des méthodes moins précises mais plus commodes. 
C'est essentiellement à la composition en grands groupes de tissus et en particulier à la détermination des tissus adipeux que les chercheurs se sont attachés. BOCCARD et DumonT (I960 $b$ ) ont dressé un tableau récapitulatif des différentes méthodes indirectes d'estimation du gras dans la carcasse. Des travaux récents tendent à montrer, que, à un âge déterminé, les proportions relatives de muscles et d'os sont peu variables, l'essentiel des variations provenant du tissu gras (KIRTON Ulyatt et Barton, I959; Tulloh, I963; Esley, McDonald, Fowler, ig64). Il en résulte qu'une détermination commode et précise de l'un de ces deux groupes de tissus (os + muscle, gras) devrait suffire à caractériser parfaitement une carcasse. Les tableaux 3, 4, 5 rapportent les principales relations calculées.

\section{TABLEAU I}

Tableau des corrélations - Section du muscle longissimus dorsi estimée-mesurée

\begin{tabular}{|c|c|c|c|c|}
\hline Auteurs & Méthode & Variable & Corrélation & $n$ \\
\hline STOUHFe et al. (1658) & Aiguille & $A+C$ & $+0,58 * *$ & \\
\hline $\begin{array}{l}\text { KNIGIIT, FoOTE, } \\
\text { BENNETT }(1959)\end{array}$ & Aiguille & Surface & $+0,53 * *$ & 36 \\
\hline \multirow{2}{*}{$\begin{array}{c}\text { CAMPBELL, STONAKER et } \\
\text { ĖSPLIN }(1959)\end{array}$} & Ultrasons & B 3 & $\begin{array}{l}+0,68 * * \\
+0,49 * *\end{array}$ & $\begin{array}{l}32 \\
33\end{array}$ \\
\hline & UItrasons & Surface & $\begin{array}{l}+0,62 * * \\
+0,4,4 *\end{array}$ & $\begin{array}{l}32 \\
33\end{array}$ \\
\hline \multirow{4}{*}{ MatTHEws et $a l .(1968)$} & $\begin{array}{l}\text { Demi-distance } \\
\text { apophyses } \\
\text { transverses }\end{array}$ & A & $\begin{array}{l}+0,15 \mathrm{NS} \\
+0,30 \mathrm{NS}\end{array}$ & $\begin{array}{l}16 \\
40\end{array}$ \\
\hline & Aiguille & B & $\begin{array}{l}+0,63 * * \\
+0,62 * *\end{array}$ & $\begin{array}{l}46 \\
10\end{array}$ \\
\hline & Aiguille & C & $\begin{array}{l}+0,57 * * \\
+0,82 * *\end{array}$ & $\begin{array}{l}46 \\
40\end{array}$ \\
\hline & $\begin{array}{l}\text { A estimé } \times \\
\text { 13 estimé }\end{array}$ & Surface & $\begin{array}{l}+0,55 * * \\
+0,69 * *\end{array}$ & $\begin{array}{l}46 \\
40\end{array}$ \\
\hline ZOBRISKY et $a l .(1961)$ & Ultrasons & Surface & $+0,80 * *$ & 70 \\
\hline 100,1 & Litrasons & Surface & $+0,21 * *$ & 82 \\
\hline DAvis et a $(150 \mathrm{t})$ & Ultrasons & C & $+0,90 * *$ & 82 \\
\hline
\end{tabular}

Signification des coefficents de corrélation (différence par rapport à 0 ) :

$* *=$ valeur hautement significative $(\mathrm{P}<0,01)$

$N S=$ valeur non significative $\left(l^{\prime}>0,05\right)$

TABLEAU 2

Liaison rendement

Pourcentage de gras dans la carcasse

(d'après Haring et al., 1954)

\begin{tabular}{l|c|c|c}
\hline Race des agneaux & Leine & Mérinos à viande & Leine $\times$ Schwarzkopf \\
& & & \\
Effectif .......... & 13 & 13 & 15 \\
P. 100 gras ....... & 8,2 a $7, \mathrm{t}$ & 7,4 & 6,5 \\
Rendement (p. 100) .. & 53,2 & 51,9 & 50,2 \\
\hline
\end{tabular}




\section{Estimation par le rendement à l'abattage.}

Le rendement d'un animal est lié en partie à l'importance de son tissu adipeux. Les résultat. épars de plusieurs auteurs, apportent quelques précisions (HANKINS et Ellis, I939; Hankins Hiner et Simmons, i95I ; Ilaring, Laydolph et Scholze, i954; Kirton et BArTon, 1962). En fait, on ne peut constater qu'une tendance de liaison entre le rendement d'une carcasse et le pourcentage de gras $(r=0,34:$ KIRTON et BARTON, 1962). Le tableau 2, extrait des résultats de IIARING, LEYDOLPI et SCIIOLZE (I954), illustre cette tendance. Une partie des variations aléatoires du rendement peut être éliminée par l'utilisation, au lieu du poids à l'abattage, du " poids vif vide " qui est le poids à l'abattage diminué du poids des contenus digestifs. On obtient alors le "rendement vrai \%.

\section{Estimation par le poids de la carcasse.}

Dans l'ensemble, les résultats obtenus à partir du poids de la carcasse sont assez décevants, les estimations étant trop peu précises pour être utiles. Les équations de prévision calculées ne semblent en outre valables que pour des animaux d'âge déterminé (BARTON et KIRTON, I958 $a$; FieLd, Kenp et VARNEY, I963).

De même l'estimation du muscle par le poids de carcasse est insuffisamment précise malgré la liaison toujours positive poids de carcasse-poids de muscle (BARTON et KIRTON, I958 $a$; AMENT, Galgan et RupNow, I962; KirTon et BarTon, 1962).

Les résultats n'étant pas plus concluants pour l'os (BARTON et KIRTON, 1958 a), il apparait que le poids de la carcasse n'apporte pas suffisamment d'informations précises sur la composition de la carcasse.

Cependant Tulloh (ig63) et Estewy, McDonald et Fowler (ig64) reprenant les résultats des travaux de Hamond, Wallace (I948), Palsson et Verges (i95o) sur les ovins, et McMeekan (I940) sur les porcs, établissent des éculations de prédiction du poids des tissus dans la carcasse, dont la forme est celle des équations allométriques de Huxley (1932). TulloII (I963) calcule ainsi trois équations en fonction du poids vif vide. Llles rendent compte respectivement de 96, I p. Ioo 98 p. Ioo et 88,3 p. Ioo des variations des tissus osseux musculaires et gras (tableaux $3,4,5$ ).

Pour tous ces travaux d'estimation de la composition de la carcasse, le choix de l'échantillon des animaux intervient sur la signification des valeurs trouvées. Lorsque les animaux étudiés sont pris au hasard quant à l'âge ou au poids, les coefficients de corrélation calculés sont beaucoup plus élevés que si l'étude porte sur un groupe homogène. Toutefois la précision de la prédiction du poids brut ou en p. Ioo de tissu n'en est pas forcément meilleure. Beaucoup de ces coefficients traduisent une tendance générale de la composition des carcasses mais le caractère mesuré ne peut servir de base à un indice d'estimation.

\section{Estimation par la masse volumique de la carcasse.}

C'est dans le domaine de l'estimation du gras dans la carcasse que la méthode de mesure par la masse volumique a été surtout utilisée. Les chercheurs se sont inspirés de travaux analogues réalisés déjà sur le cobaye, le rat albinos et le porc. Cependant les conditions normales sont trop difficiles à réaliser avec un animal de la taille dı mouton : au cours de l'immersion des carcasses, il est impossible de parvenir à éliminer tout l'air accumulé dans les cavités ou adhérant aux tissus. Aussi les coefficients de corrélation linéaire trouvés, bien qu'assez élevés, ne permettent-ils pas une bonne estimation du tissu gras. Les résultats obtenus avec les équations de prévision présentent un écart-type résiduel trop important (tabl. 3). CowAn et al. (Ig61) font les mêmes remarques à la suite de travaux portant sur la détermination de la valeur énergéticue de la carcasse à l'aide de la même méthode.

\section{Estimation par la méthode au potassium-40}

Le potassium est le cation le plus important en pourcentage dans le tissu musculaire. La double hypothèse d'une proportion constante de potassium dans le tissu maigre d'une part, et d'une proportion constante de l'isotope ${ }^{40} \mathrm{~K}$ radioactif dans le potassium naturel d'autre part, constitue la base théorique de la méthode utilisée. Il est intéressant d'établir une relation étroite entre la quantité de rayons émis en un temps donné par le ${ }^{40} \mathrm{~K}$ d'une carcasse et le pourcentage de tissu musculaire et des autres tissus de cette carcasse. Cette méthode présente l'énorme avantage d'une utilisation sur animal vivant (KIRTON et al., 1961; Jubge et al., 1963). Cependant laz précision n'atteint pas le niveau que pouvait laisser présager la théorie de la méthode. Lt JUDGE et al. (1963) indiquent même que les résultats ne sont pas plus satisfaisants qu'en utilisant d'autres mesures telles celle de la surface de la section du muscle long dorsal (corrigée pour un poids de carcasse de $100 \mathrm{lb}$ ). 
J. C. FLAMANT, R, BOCCARD

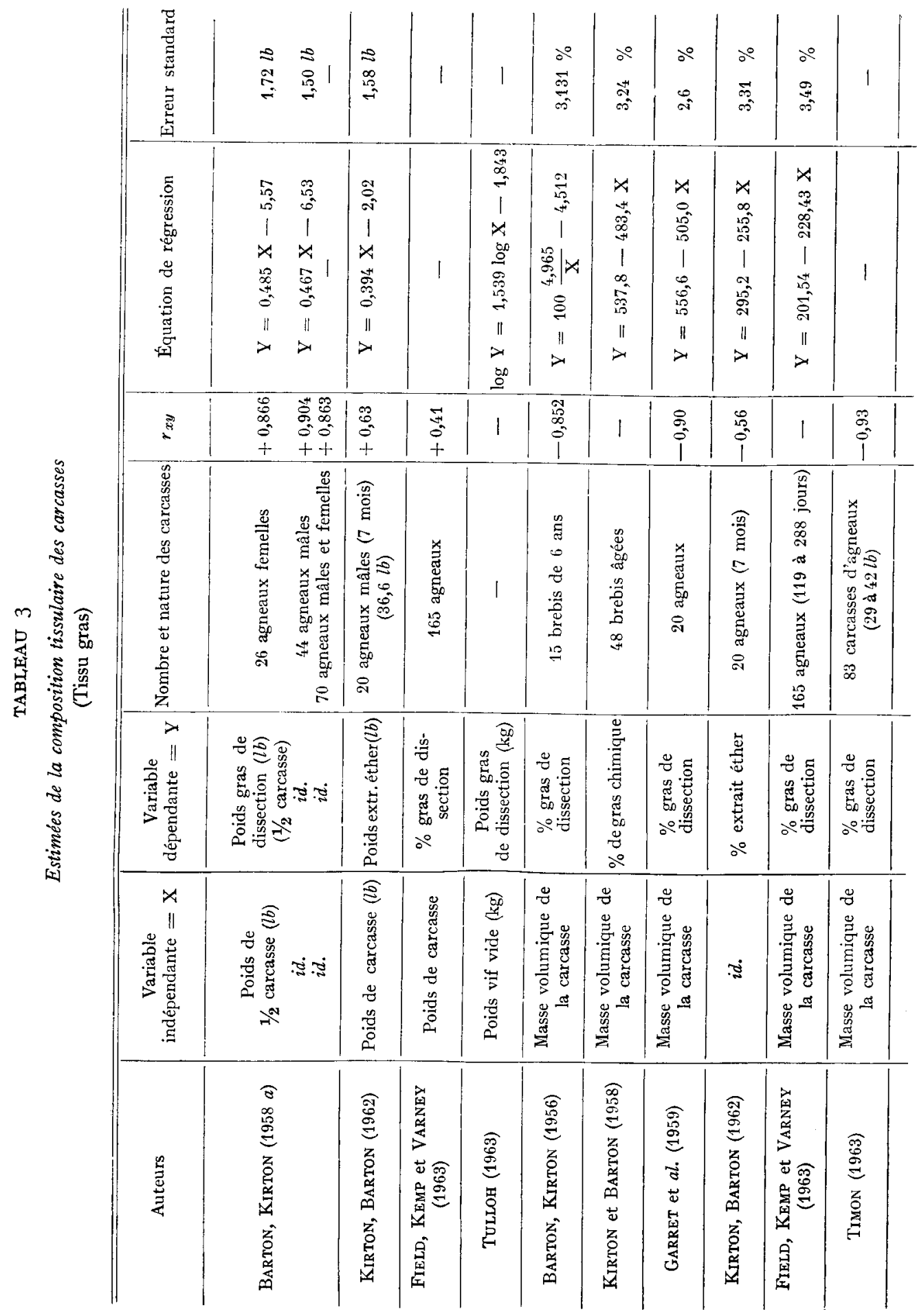




\begin{tabular}{|c|c|c|c|c|c|c|c|c|c|c|c|}
\hline 1 & $t$ & $\begin{array}{l}\circ \\
\stackrel{9}{\circ} \\
\infty\end{array}$ & $\begin{array}{l}\circ \\
\infty \\
\infty \\
\infty \\
\infty\end{array}$ & $\stackrel{20}{\stackrel{2}{\pi}}$ & 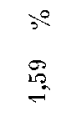 & i̊ & 1 & 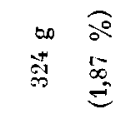 & $\begin{array}{l}\circ \quad 0 \\
\vdots\end{array}$ & 1 & 11 \\
\hline 1 & 1 & 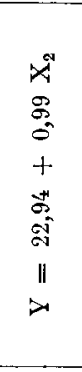 & 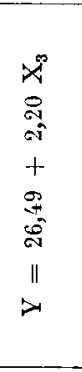 & 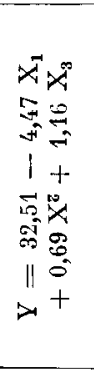 & $\begin{array}{l}8 \\
0 \\
+ \\
+ \\
x \\
2 \\
0 \\
0 \\
11\end{array}$ & \begin{tabular}{l}
8 \\
\multirow{2}{*}{} \\
+ \\
$x$ \\
$x$ \\
2 \\
0 \\
11 \\
$>$
\end{tabular} & 1 & 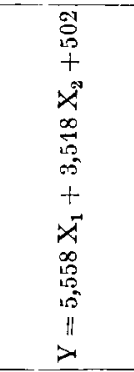 & 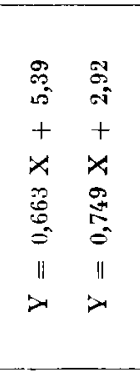 & 1 & 1 \\
\hline $\begin{array}{l}8 \\
0 \\
+ \\
+\end{array}$ & 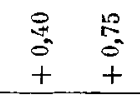 & $\begin{array}{l}8 \\
8 \\
0 \\
+\end{array}$ & $\begin{array}{l}\text { 号 } \\
0 \\
+ \\
+ \\
\end{array}$ & 1 & $\begin{array}{l}\infty \\
0 \\
0 \\
+ \\
\end{array}$ & 1 & $\begin{array}{l}8 \\
8 \\
+ \\
+\end{array}$ & 1 & $\begin{array}{l}5 \\
0 \\
0 \\
0 \\
+ \\
\end{array}$ & $\begin{array}{l}\mathscr{0} \\
\stackrel{5}{0} \\
\dot{0} \\
+\quad+\end{array}$ & $\begin{array}{ll}8 & 0 \\
0 & \infty \\
0 & 0 \\
+ & + \\
\end{array}$ \\
\hline 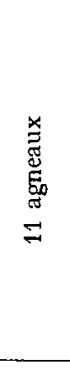 & 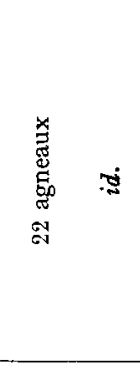 & 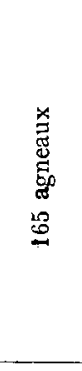 & 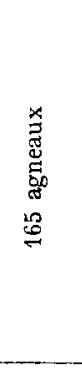 & : & 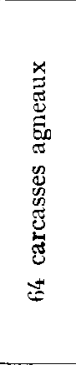 & - & 1 & 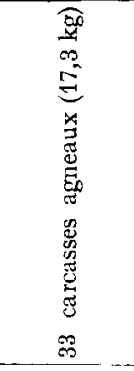 & 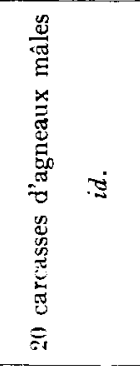 & 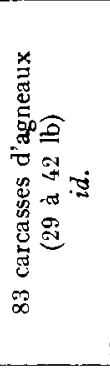 & 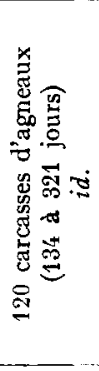 \\
\hline 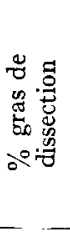 & 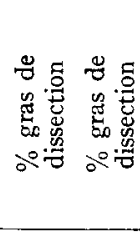 & 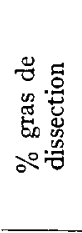 & 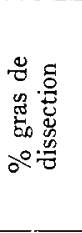 & . & 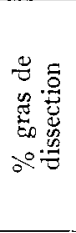 & 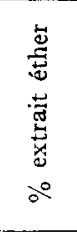 & 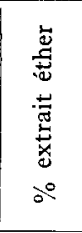 & 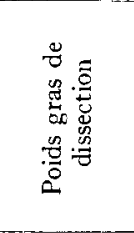 & 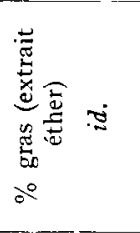 & 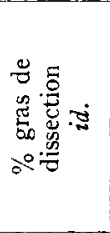 & 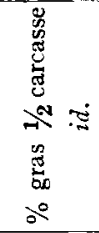 \\
\hline 0 & 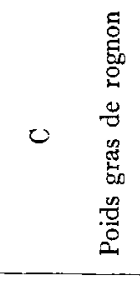 & $\begin{array}{c}0 \\
11 \\
x^{N}\end{array}$ & 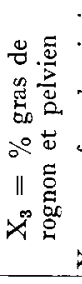 & 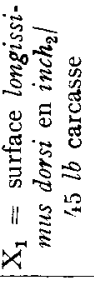 & 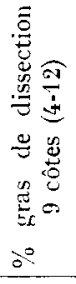 & 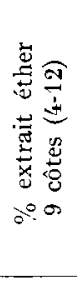 & 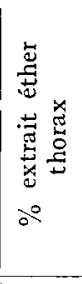 & 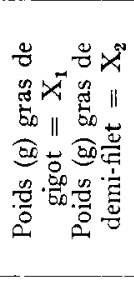 & 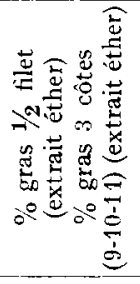 & 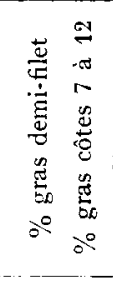 & 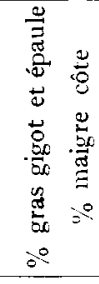 \\
\hline 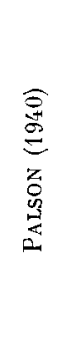 & 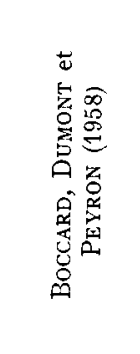 & & 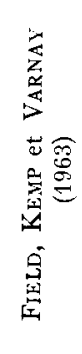 & & (2) & $\begin{array}{l}\frac{E}{2} \\
\frac{\pi}{2} \\
\frac{n}{2} \\
\frac{\pi}{2}\end{array}$ & 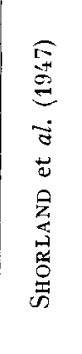 & 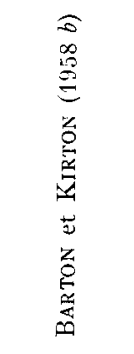 & 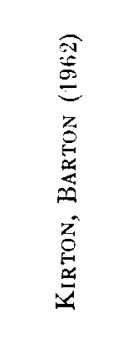 & 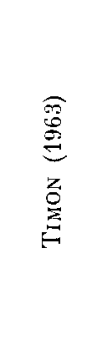 & 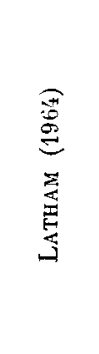 \\
\hline
\end{tabular}




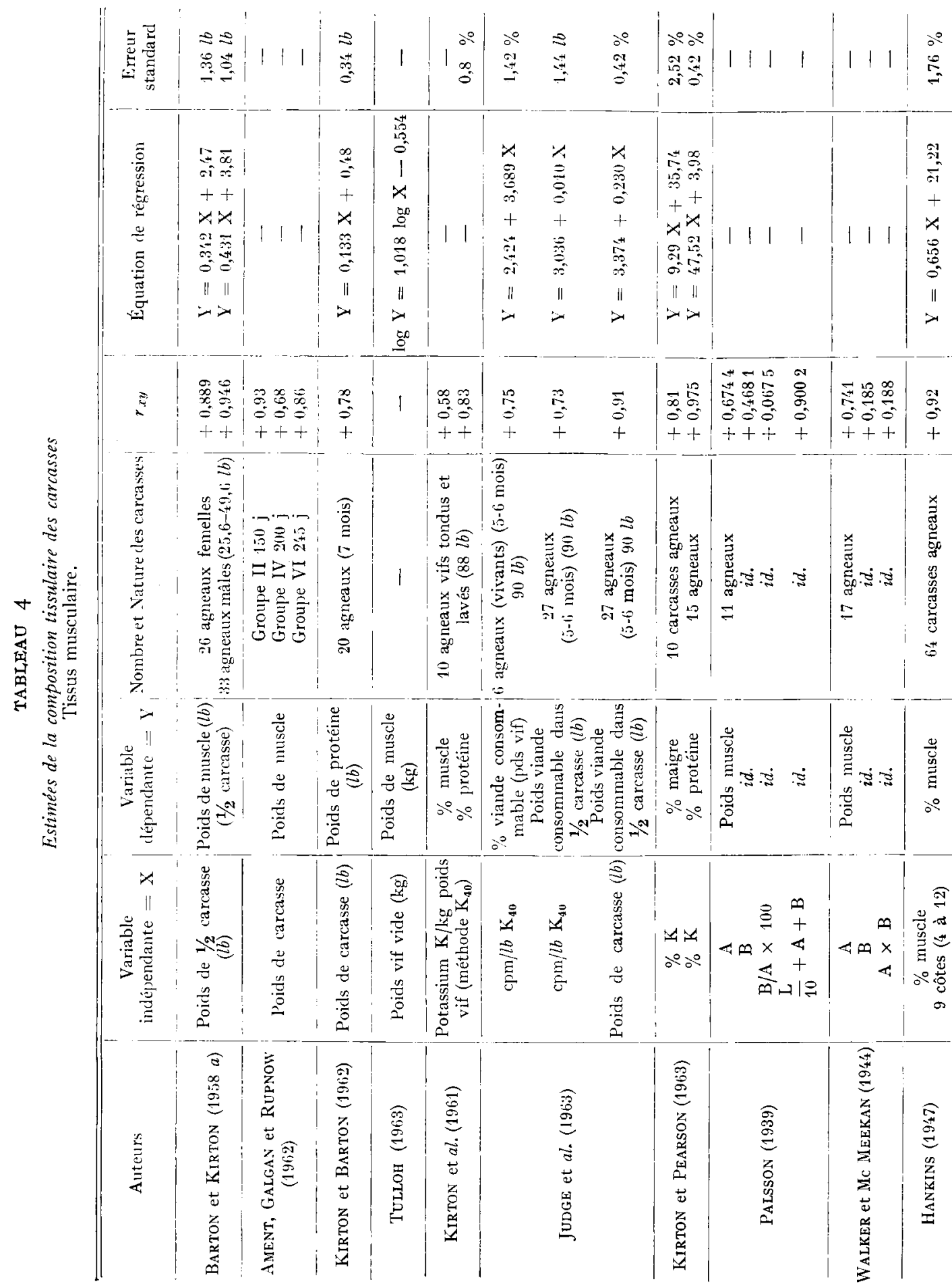


Kauffuan et Norton (I964) trouvent de grandes variations de la teneur en potassium naturel entre les muscles de diverses régions anatomiques. Cependant la liaison entre la teneur en potassium, mesurée au photomètre de flamme, et les pourcentages d'eau, d'extrait à l'éther et de protéine dé la carcasse est très élevée. La précision de la prédiction est nettement meilleure que celle obtenue par l'utilisation du potassium-40 (KIRTON et PEARSON, I963), et peut donc servir d'étalon aux méthodes améliorées de mesure par ${ }^{40} \mathrm{~K}$ sur animal vif (tabl. 4 ).

\section{Estimation à l'aide de mesures linéaires diverses.}

Les mesures de conformation effectuées sur les carcasses peuvent constituer des mesures d'estimation de la composition tissulaire. Si des liaisons suffisamment étroites sont trouvées, on dispose d'un indice dont l'utilisation est facile. l)es carcasses peuvent alors être classées pour leur composition en maigre ou gras sans être mutilées ou rendues impropres à la commercialisation. L'étude de ces relations permet en outre de déterminer dans quelle mesure la conformation et la composition sont liées.

\section{a) Muscle (tabl. 4).}

La littérature rapporte de nombreux calculs de coefficients de corrélation, de valeur plus ou moins forte, de diverses combinaisons de mesures et de poids avec le pourcentage ou le poids de muscle dans la carcasse. 11 semble en fait yue les mesures utilisées, et en part iculier certaines mesures de forme de la carcasse, ne soient (ju'une estimation du poids de la carcasse, facteur déterminant pour beaucoup le poids du tissu musculaire total : ceci expliquerait que leur valeur de prédiction de la composition tissulaire soit inférieure ou au plus égale ì celle obtenue par l'utilisation du poids de carcasse. 'Toutefois on peut regretter que les auteurs n'aient pas testé la signification des nouveaux critères lorsqu'on a déjà tenu compte de l'influence du poids de carcasse. L'estimation de la composition par les mesures homologues sur animal vif n'apporte évidemment pas de précision supplémentaire.

Boccard, Dumont et Priron (i958) ont dressé un tableau des résultats les plus significatifs. Ils soulignent la faible liaison existant entre l'épaisseur du longissimus dorsi et le poids total de muscle, la corrélation négative pour l'indice $\frac{\mathrm{L}}{\mathrm{IO}}+\mathrm{A}+\mathrm{B}$ de P'ALSson (I939) qui présente la liaison la plus étroite mais dont la détermination nécessite une découpe de la carcasse. Ia forme de la section du muscle longissimus dorsi, souvent considérée comme indice de développement musculaire, est donc sans liaison étroite avec le poids total de muscle. Nous ne pouvons donc considérer le critère "forme de la noix de côtclette "que comme une coquetterie de conformation, particulière uniquement à ce morceau. Botkin, Stanley et Schoonover (1959). Knigit, Foote et BennetT (i 959), Ament Galgan et RupNow (1952), Field, Kemp et VARNiy (1963), Judge et al. (1963), déterminent des liaisons étroites entre la surface de la section du longissimus dorsi et le muscle total de la carcasse. Ces coefficients assez élevés ne dépassent pas ceux obtenus avec le poids de la carcasse, et sont encore beaucoup plus faibles si des corrections sont faites pour le poids de la carcasse qui détermine fortement la surface de la section : Esplin, Henry et Sutilerlani) (ig64), Bailey, Pople et Cirapman (196I). Il semble donc que les mesures d'extérieur de la carcasse et éventuellement sur le longissimus dorsi, ne permettent pas une estimation valable du tissu maigre de la carcasse.

La pesée de muscles individuels ne permet pas d'obtenir une précision supplémentaire, les corrélations calculées étant du même ordre que celles obtenues avec la surface du longissimus dorsi (Ament, Galgan et Ruinow, 1962 ; OrMe, Citristian et Bell, i962). C'est pour tenir compte du rôle important du poids que JUDGE et MARTIN (196.3) proposent une équation de régression multiple où interviennent le poids de la carcasse et 2 mesures de gras (épaisseur du gras dorsal et poids du gras de rognon) pour estimer la proportion de viande consommable.

\section{3) Os (tabl. 5).}

Palsson (1940) remarque que les races de moutons (qu'il étudie sont classées dans le même ordre pour le poids moyen du squelette de leurs représentants et pour le poids du canon avant gauche $\grave{a}$ âge et à poids de carcasse constants chez les agneaux et less adultes.

S'inspirant de travaux précédents et après avoir comparé leurs propres résultats à ceux de Palsson (I939-1940) et ì ceux de Waiker et McMeekan (I944), Boccard Dimont et Peyron (1958) proposent pour estimer l'importance du tissu osseux d'une carcasse :

- le poids du (ou des) canon (s),

- ou un indice "os ", quand l'os canon fait défaut, constitué par la moyenne des sommes des 2 mesures suivantes effectuées sur les deux côtés de la carcasse à l'aide d'un pied à coulisse:

- distance entre les malléoles internes du tibia et la base du calcanéum. 
- distance entre le bord externe des os cuboïdoscaphoïdien et du grand cunéiforme.

ULYATT et BARTON (1963) préfèrent utiliser la relation os canon-os total qui, bien qu'encore insuffisante en précision est plus étroite que celle obtenue par l'utilisation de méthodes chimiques étudiées par ailleurs.

\section{TABLEAT 5}

Estimation de la composition tissulaire des carcasses

(Tissu ossenx)

\begin{tabular}{|c|c|c|c|c|c|c|}
\hline Auteurs & $\begin{array}{l}\text { Variable } \\
\text { indépendante } \\
=\mathrm{X}\end{array}$ & $\begin{array}{l}\text { Variable } \\
\text { dépendante } \\
=Y\end{array}$ & $\begin{array}{l}\text { Nombre et } \\
\text { nature des } \\
\text { carcasses }\end{array}$ & $r_{x y}$ & $\begin{array}{l}\text { Équation } \\
\text { de } \\
\text { régression }\end{array}$ & $\begin{array}{l}\text { Erreur } \\
\text { standard }\end{array}$ \\
\hline $\begin{array}{l}\text { Barton et Kirtoni } \\
\left(\begin{array}{lll}1958 & a\end{array}\right)\end{array}$ & $\begin{array}{l}\text { Poids de } 1 / 2 \\
\text { carcasse }(l b)\end{array}$ & $\begin{array}{c}\text { Poids os } \\
(1 / 2 \text { carcasse })\end{array}$ & 3.3 agneaux máles & $+0,794$ & $\begin{array}{c}Y=0,076 \mathrm{X} \\
+1,80\end{array}$ & $0,41 \quad l b$ \\
\hline PalsSON (19:36) & $\begin{array}{c}\text { Poids canon } \\
\text { antérieur gauche }\end{array}$ & Poids os & 11 agneaux & $+0,9432$ & & \\
\hline $\begin{array}{c}\text { Boccard, DUMONT } \\
\text { et PEyRON }\end{array}$ & $\begin{array}{l}\text { Poids canon } \\
\text { antérieur }\end{array}$ & Poids os & 155 agneaux & $+0,9178$ & & \\
\hline & Indice " os & Poids os & 153 agneaux & $+0,7166$ & & \\
\hline $\begin{array}{c}\text { ULYATT Et BARTON } \\
(1963)\end{array}$ & $\begin{array}{l}\text { Poids canon } \\
\text { antérieur }(l b)\end{array}$ & Poids os $(l b)$ & 39 brebis (2 ans) & $+0,821$ & $\begin{aligned} Y & =0,15 X \\
& +0,51\end{aligned}$ & $0,44 l b$ \\
\hline Tulloh (1963) & $\begin{array}{l}\text { Poids vif vide } \\
\qquad(\mathrm{kg})\end{array}$ & Poids os (kg) & -- & - & $\mid \begin{array}{l}\log Y \\
\log X\end{array}$ & \\
\hline HANKINS $(1917)$ & \begin{tabular}{|c|} 
p. 100 cendres \\
dans 9 côtes \\
$(4$ à 12$)$ \\
id. Epaule \\
id. Gigot \\
$\%$ os + ligament \\
dans 9 cotes
\end{tabular} & $\begin{array}{c}\text { p. } 100 \text { cendres } \\
i d . \\
i d . \\
\% \text { os } \\
+ \text { ligament }\end{array}$ & $\begin{array}{l}35 \text { agneaux } \\
\quad i d . \\
\quad i d . \\
\text { bit agneaux }\end{array}$ & $\begin{array}{l}+0,73 \\
+0,89 \\
+0,81 \\
+0,97\end{array}$ & $\mid \begin{array}{l}\mathbf{Y}=0,80 \mathrm{X} \\
+0,15 \\
\mathbf{Y}=0,78 \mathrm{X} \\
+3,96\end{array}$ & $\begin{array}{l}0,05 \% \\
1,68 \%\end{array}$ \\
\hline
\end{tabular}

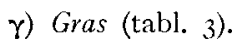

Certaines liaisons, notamment parmi celles déterminées par Hankins, Hiner et Simmons (I9II), FIELD, KEMP et VARNEY (I963), sont trop faibles pour présenter un réel intérêt pratique pour l'estimation du gras dans la carcasse (longueur moyenne du corps, surface de la section du muscle long dorsal).

Par contre, on obtient des liaisons plus étroites par la mesure de l'épaisseur du gras au-dessus du longissimus dorsi ( $\left(2^{2}\right.$ côte, mesure C) (PALsson, I940; BOcCard, DUMONT et PEYron, 1958) ou par la pesée du gras de rognon (Hankins, IIIner et Simmons, I95I ; Boccard Dumont et PEYRON, I958; BoCCARD et 1)UMONT, I960; Field, Kemp et VARNeY, 1963). D'autres mesures ont été définies par Palsson et sont rapportées par BocCard, Dumont et Peyron (1958). Cependant les précisions obtenues ne semblent pas toujours bonnes. Ces mesures paraissent mieux convenir pour différencier entre eux des groupes de carcasses que comme index de composition (AMENT, Galgan et RupNow, ig62).

Field, KeMp et VARNEY (1963) établissent une équation de prédiction (régression multiple) nécessitant la mesure de la surface de la section du muscle long dorsal, la mesure $\mathrm{C}$ et le poids du gras de rognon. La nécessité de la découpe et la complexité de l'équation ne sont pas compensées par un gain appréciable de précision.

Pour l'ensemble des estimations indirectes de la composition des carcasses on peut critiquer 
l'utilisation des rapports qui peut introduire des corrélations "fallacieuses " n'ayant pas forcément un sens biologique, du type corrélation entre $y$ et $\frac{x}{y}$. La formule mathématique de la corrélation entre $y$ et $\frac{x}{y}$ connaissant les paramètres de la loi du couple $(x, y)$ a d'ailleurs été obtenue et DıNKEL et al. (1965) ont publié une critique de l'utilisation des rapports et pourcentages comme indices dans l'étude des carcasses. Il semble que les travaux de prédiction seraient utilement repris par l'utilisation des méthodes de régression linéaire ou curvilinéaire classiques.

Estimation par la composition des morceaux de la carcasse (tabl. 3, 4, 5).

La plupart des auteurs dont nous avons rapporté les travaux dans les paragraphes précédents ont analysé des demi-carcasses. CARroll et O'CARROLL (1964) montrent que l'analyse d'une demicarcasse ne diminue pas la précision de l'estimation de la composition de la carcasse entière de façon considérable sauf pour l'os où les variations de découpe au niveau de la colonne vertébrale introduisent un manque de précision de la méthode. Les auteurs proposent de maintenir entière la colonne vertébrale qui représente 29 p. Ioo de l'os total.

Mais au lieu de procéder à une dissection complète de la carcasse ou d'une $\mathbf{I} / 2$ carcasse, il est plus rapide et économique de n'étudier qu'un seul morceau, à condition qu'il reflète particulièrement bien la composition de l'ensemble de la carcasse. La méthode utilisée doit chaque fois faire appel à une découpe normalisée pour l'isolement des morceaux. L'analyse peut être physique (isolement des tissus), ou chimique en utilisant les relations existant entre muscle et matière azotée, gras et extrait à l'éther, os et cendres, respectivement (ShORLAND et al., i947., UlyatT et BarTon, i963) : les liaisons les plus élevées sont obtenues pour le gras et les plus faibles pour l'os. L'analyse chimique présente en outre l'intérêt de permettre une détermination de la valeur énergétique des carcasses.

Que l'analyse soit physique ou chimique, plusieurs auteurs anglo-saxons (HaNkINS, I947 ; Shorland et al., 1947; BarTon, Kemp et VARney, 1963; Timon, 1963) estiment que la facilité relative de découpe des régions dorsales (côte ou filet) constitue un avantage déterminant sur les autres morceaux (présentant eux aussi des liaisons étroites de composition avec l'ensemble de la carcasse). KIRTON et BARTON ( $\mathrm{Ig62}^{6}$ ) soulignent qu'un morceau parfaitement défini par la découpe, tel le demi-filet droit. est de petite dimension et facilite ainsi les manipulations tout en permettant une bonne précision de prédiction.

Ces résultats ne peuvent toutefois être transposés directement en France.

$\mathrm{I}^{\mathrm{O}}$ Les côtes ou le filet ne sont pas les morceaux les plus faciles à isoler de la carcasse sans forte dépréciation de la carcasse.

$2^{\circ}$ Il est possible d'utiliser d'autres morceaux dont la composition est en liaison étroite avec celle de la carcasse (gigot, épaule...) (LATHAM et al., 1964).

Il est donc souhaitable que de tels travaux soient repris avec les morceaux de la découpe française afin de déterminer si un morceau tel que l'épaule ne serait pas d'utilisation plus commode.

Ces méthodes d'estimation nécessitant une découpe partielle, constituent un stade avancé de la connaissance de la composition des carcasses. En général elles ne sont pas utilisables dans la pratique en dehors des travaux de recherche, les carcasses étant notablement dépréciées par les prélèvements effectués. Il s'agit donc de méthodes de laboratoire.

De toutes les méthodes rapportées ici, beaucoup d'entre elles, en dépit de leur simplicité, ne sont promises à aucune exploitation pratique. Nous devons donc éliminer certaines mesures de forme de la carcasse, et aussi les déterminations de masse volumique. Les renseignements qu'elles nous apportent sur la composition en tissus des carcasses sont trop grossiers. Le poids de la carcasse ne peut être considéré seul mais en association avec d'autres mesures. La région dorsale se révèle être un indice de composition acceptable mais la méthode nécessite une découpe et une dissection délicates. En dépit de la bonne précision de leur estimation, les méthodes chimiques de détermination de la composition en tissus présentent l'inconvénient, comme les méthodes de dissection, de diminuer la valeur commerciale des carcasses par suite des mutilations qu'elles entraînent. La méthode au ${ }^{40} \mathrm{~K}$ qui suscitait au départ de grands espoirs ne les a pas confirmés jusqu'à présent. Son étude expérimentale doit se poursuivre. Son utilisation possible sur animal vif présente le plus grand intérêt malgré le coût élevé de l'appareil nécessaire.

Les meilleurs indices actuels de composition tant par leur précision que par leur facilité d'obtention sont le poids de l'os canon pour l'appréciation du tissu osseux, et le poids du gras de rognon associé à l'épaisseur du gras de couverture pour l'appréciation du tissu gras. Ces mesures sont facilement déterminables moyennant certaines précautions prises dans le traitement des carcasses immédiatement après l'abattage. Elles doivent permettre un classement rapide pour la composition tissulaire. Une étude de la composition des carcasses pourrait donc comprendre tout d'abord une éva. 
luation des tissus gras et osseux à l'aide de ces deux indices. Une méthode plus élaborée consisterait à utiliser la liaison entre la composition de la carcasse et celle des morceaux. Le filet ou les côtes pourraient être utilisés comme dans les travaux anglo-saxons, mais l'épaule, dont la découpe ne présente pas de difficulté majeure en France, mériterait une attention particulière.

Il semble par ailleurs que nous soyons encore loin de posséder une estimation précise de la composition du corps à partir de l'animal vif. La revue bibliographique effectuée récemment par KIRTON (I964) nous indique que la mesure idéale n'est pas encore au point. Jusqu'à présent les mesures linéaires de conformation, le poids du corps, les mesures linéaires internes (épaisseur du gras), la masse volumique (dans un fluide liquide ou gazeux) et l'estimation au potassium-4o n'ont pas constitué des indices valables. Une technique originale consiste à évaluer la composition en eau du corps et à en déduire le poids du maigre + os (fat free body). La base théorique de cette méthode est l'hypothèse que le poids du corps sans le gras est constant pour un animal d'un âge donné dans une espèce donnée. Il suffit de déterminer directement le volume d'eau du corps à l'aide d'une " substance marqueuse " (antipyrine et dérivés) (DUMONT, I955), et de se servir de la relation supposée fonctionnelle entre l'eau du corps et les tissus musculaires et osseux. Le poids du tissu gras peut être déterminé ensuite par différence. La mise au point d'une méthode valable permettrait en particulier l'estimation de la composition tissulaire et chimique du corps de l'animal au cours de sa croissance.

\section{IV. - DISCUSSION}

Nous avons passé en revue diverses méthodes de mesures dont l'objectif est une meilleure connaissance des carcasses:

- connaissance très fine au niveau de sa composition chimique, tissulaire ou par morceaux et régions anatomiques ;

- indices de composition nécessitant une découpe mais évitant des dissections, longues et coûteuses dans les travaux expérimentaux;

- indices de compositions plus grossiers mais d'utilisation possible plus routinière moyennant des règles simples;

- mesures de conformation de la carcasse et indices de forme (gigot, muscle, long dorsal);

- mesures de jugement de la conformation et évaluation de la composition de la carcasse sur animal vif, susceptibles d'une utilisation en sélection massale.

Munis de ces instruments de travail nous pouvons nous demander quels sont effectivement les critères directeurs intervenant dans le jugement habituel des carcasses. Ce chapitre rassemble diverses réponses à cette question, nous renseignant sur les relations susceptibles d'exister entre les mesures objectives sur carcasses et la qualité de ces mêmes carcasses appréciées sur le marché.

Pour juger valablement l'intervention des différents critères de qualité d'une carcasse il ne suffit pas de constater des relations entre le classement des carcasses et différentes mesures. On peut en effet se demander si le classement commercial reflète bien la qualité des carcasses c'est-à-dire la demande du consommateur. Un certain nombre de travaux indiquent en effet que le prix payé par unité de poids de carcasse peut être un indice beaucoup plus sensible des orientations du marché. Une distorsion entre la classe de qualité de carcasse et les cours peut intervenir dans un marché en évolution (U.S.A.) : Phillips, CARD et CARTER (1963) vérifient que sur le marché de New York des carcasses Prime ont dû être déclassées et vendues moins cher par suite du peu d'empressement des acheteurs pour des carcasses en moyenne trop lourdes et trop grasses.

\section{Relation poids-qualité des carcasses}

Des résultats très divers ont été rapportés par les auteurs concernant l'influence du poids sur la qualité des carcasses. Cette diversité ne fait que refléter la variété de la demande pour le poids des carcasses d'un pays ou d'une région à l'autre. Les travaux d'auteurs américains (KEMP, Bull et BEAN, 1953) permettent de constater que le classement pour la qualité commerciale correspond aussi en moyenne à un classement pour le poids. Phillips, CARD et Carter (I960) indiquent que dans le Kentucky les agneaux Prime sont en moyenne les plus lourds.

En Australie, la répartition des carcasses en classes tient compte explicitement à la fois du poids et de la conformation (RoBinson, BINET et DoIG, 1956). I ua situation est la même en NouvelleZélande (ClaRke et McMeEKan, I952). Aussi constate-t-on que les carcasses les plus lourdes tendent à obtenir les meilleures notes, ce qui se traduit par des corrélations positives (en supposant que les notes les plus élevées sont attribuées aux carcasses les meilleures) : Neville, ChAPMAN et POPE (I958) $(r=0,55$ à I $20 \mathrm{j}$.), SKJERVOLd et GJEDREM (I958) $r=0,75$ à I65 j). La même tendance, 
semble se manifester dans les jugements sur animaux vivants : TANEJA (I955 b) $(r=+0,44$ à 17 mois, $r=+0,4 \mathrm{I}$ à I I mois, $r=0,26 \grave{a} 5$ mois). Les carcasses les plus lourdes étant en moyenne plus grasses, du moins à l'époque de ces travaux, pourraient bénéficier d'un jugement plus favorable dans le cas des marchés anglo-saxons (RoBinson, Binet et Iorr, 1956). A l'appui de cette thèse on peut citer les travaux de DF: BACA et BOGART (I 959) qui trouvent une relation entre la note de conformation et l'étal d'engraissement. Cependant PintLlips, Card et Carter (ig6o) et CarpenTIER et al. (1964) déterminent une relation négative entre le prix payé par livre de carcasse et le poids de ces carcasses.

\section{Retation conformation-qualité des carcasses}

La conformation est considérée comme un des critères principaux de la qualité des carcasses. La raison en est la notion implicite et largement partagée qu'elle détermine la composition des carcasses.

IIUNDT (1959) remarque que la note de conformation des carcasses, jugées selon l'échelle essentiellement subjective de la I. L. G. (Deulsihe Landrivitschaftiche Gesellschaft), s'accroît lorsque la profondeur de poitrine relative (profondeur de poitrine/hauteur de garrot) augmente jusqu’à $47,5 \mathrm{p}$. Ioo. Lal note de conformation de la carcasse, du filet et du gigot s'éleve aussi lorsque le bassin est plus large. Wais il n'y a pas de relation nette entre la note d'appréciation de la carcasse et la longueur du corps.

Il est intéressant de rechercher, après classement subjectif des carcasses, quelles sont les mesures qui en rendent le mieux compte, c'est-ì-dire les mesures qui différencient le mieux les carcasses en présence. Dans ce but BRIER, ScHotT et SinMons (1940) étudient une méthode d'établissement de fonction discriminante sur le mouton. RoBINSON, BINET et Dorg (I956) proposent une fonction discriminante établie à partir de 10 mesures effectuées sur des carcasses d'agneaux austra. liens. Ies carcasses sont classées selon le systeme adopté dans ce pays :

Doz'n Royal : carcasses d'excellente conformation issues d'animaux de type Down.

Royal : carcasses d'excellente conformation appartenant it d'autres races.

Tallarook, et liir : carcasses de moins bonne conformation on maigres.

L'étude porte sur 240 carcasses après une étude préliminaire portant sur 50 carcasses. Ces carcasses appartiennent à plusieurs classes de poids.

$\begin{array}{llllll}\text { light } & 27 & \text { aे } & 28 & \mathrm{lb} \\ \text { medium } & 35 & \mathrm{a} & 36 & \mathrm{lb} \\ \text { heary } & 43 & \mathrm{a} & 4 & 4 & \mathrm{lb} \\ \text { overall } & 5 \mathrm{a} & \mathrm{a} & 52 & \mathrm{lb}\end{array}$

La meilleure discrimination entre les classes de carcasse pour la conformation est obtenue avec les mesures suivantes:

$$
\begin{aligned}
& \text { classe light : F, K, BC, Sh, P. } \\
& \text { classe medium: } \mathrm{F}, \mathrm{Th} . \\
& \text { classe heavy }: \mathrm{F}, \mathrm{K}, \mathrm{W}, \mathrm{P} . \\
& \text { classe overall : } \mathrm{F} \text {. Th, BC, P. }
\end{aligned}
$$

$\mathrm{K}$ est la longueur de la carcasse

Sh l'épaisseur de l'épaule

W la largeur de l'épaule

F représente la longueur du gigot (crosse-périnée)

Th la profondeur du thorax

$\mathrm{BC}$ l'épaisseur du filet (long dorsal + gras)

$P$ l'épaisseur du flanc.

Nous devons constater la très grande importance prise par la mesure F (longueur crosse-périnée) qui constitue ainsi un excellent index de conformation valable pour toutes les classes de carcasses en présence. La fonction discriminante proposée et utilisable comme index de conformation est la suivante pour toutes classes de poids :

$$
y^{\prime}=0,057 \mathrm{~F}+0,050 \mathrm{Th}-0,097 \mathrm{BC}-0,17 \mathrm{P}
$$

Une note de conformation peut ensuite être attribuée pour chaque carcasse :

$$
y=250-10 y^{\prime}
$$

Annales de Zootechnie. - ig66. 
HUNDT (1959) après avoir étudié diverses relations sur les carcasses d'agneaux "Tête noire " (Schwarzkopf) et Mérinos à viande, pense pouvoir définir l'animal donnant la carcasse de meilleure qualité de la façon suivante :

\author{
hauteur au garrot $6 \mathrm{r} \mathrm{cm}$ \\ profondeur de poitrine relative 49,4 p. 100 \\ largeur de poitrine $27,5 \mathrm{~cm}$ \\ largeur du filet $29 \mathrm{~cm}$ \\ longueur du corps $69 \mathrm{~cm}$.
}

L'appréciation de la conformation intervient toujours dans la note de qualité attribuée. Cependant les différences entre classes de carcasses dues à certaines mesures particulières " de conformation " sont difficiles à mettre en évidence. Ainsi Clarke et Mc MeEKan (1952) comparent les agneaux néo-zélandais classés dans l'ordre Down, Prime et Second. Les agneaux Down ont un membre arrière en moyenne plus court que les agneaux l'rime. Mais la largeur du gigot G est de même importance dans les 2 classes, et les agneaux Second ont une largeur de gigot G identique.

En fait, pour beaucoup d'auteurs, la qualité d'une carcasse se confond avec la conformation de cette carcasse. Des différences nettes entre classes de qualité de carcasses se manifestent surtout à propos des mesures de longueur des rayons osseux. Un canon court et épais avec des extrémités relativement fines paraît être associé à une très bonne qualité de carcasse (P'ALSSON, I940) toutes choses égales par ailleurs. Pour Clarke et Mc Mrekan (1952) les carcasses les mieux classées paraissent correspondre effectivement à des membres courts.

Bien que ces travaux nécessitent en général la découpe des carcasses (les autres méthodes de mesures étant encore en cours d'étude -- cf. $2-b$ Ch. III) nous rapportons dans ce paragraphe les résultats relatifs à la forme de la section du muscle long dorsal. En général, les carcasses de classe plus élevée paraissent avoir un muscle long dorsal plus épais et moins large (rapport B/A plus élevé) (Clarke et McMeskan, i952). Cette liaison s'exprime par des corrélations cependant assez faibles, calculées entre les dimensions de la section et la note de qualité (Neville, Pope et Crapman, i958, MatTEws et $a l .$, ig60). Les valeurs trouvées sont seulement significatives pour A (section d'autant plus longue que la qualité est basse, à poids constant). Il se confirme ici que ce facteur "forme de la section du long dorsal" n'est qu'une caractéristique très particulière à la noix de côtelette, beaucoup moins importante que chez le porc ou le boxuf, sans répercussion notable sur la qualité des carcasses, et de plus sans liaisons très étroites avec le muscle total de la carcasse. Les essais de détermination de la surface de la section du muscle long dorsal par des méthodes compliquées et coûteuses paraissent donc assez vains, bien que PALSSON (1950) et PALSSON et VERges (1950) semblent at tacher une certaine importance à l'association entre un canon court et un muscle long dorsal épais, et que Palsson (1940) détermine des liaisons très étroites entre la flèche de la côte (exprimée en pourcentage de la longueur de la côte) et les mesures $\mathrm{B}(r=+0,82)$ et $\mathrm{B} / \mathrm{A} \times 100(r=+0,68)$ calculées sur I I carcasses. (La flèche de la côte représente en fait la largeur de la carcasse).

Ces quelques travaux mettent en lumière la très grosse influence de la conformation sur le jugement de la qualité des carcasses. La longueur des rayons osseux et singulièrement la longueur des gigots sont les facteurs déterminants, les autres éléments de la conformation généralement invoqués ne paraissant pas intervenir de façon sensible dans la note finale.

\title{
3. Relation composition en morceaux-qualité
}

Mis à part le critère de la conformation, on a souvent invoqué l'importance des morceaux de ${ }_{\text {I }}^{\text {re }}$ catégorie pour définir la qualité des carcasses. Aussi beaucoup d'auteurs ont-ils déterminé le pourcentage de ces morceaux et ont désiré exprimer la liaison avec la qualité de la carcasse, pensant la trouver positive conformément à l'opinion courante. Or, cette association entre classes de carcasse et résultats de la découpe n'est pas aussi satisfaisante qu'on le souhaiterait (ZoBRISKY et al. I96r). Boccard et Dumont (r960 a) ont émis l'hypothèse de l'existence d'une " harmonie anatomique " qui, à même poids, et même état d'engraissement entraînerait une constance de l'importance relative des régions corporelles, indépendante de la morphologie extérieure des agneaux.

Cependant KEMP, Bull et BEAN (1953), sur 40 agneaux Western, constatent une liaison faible du pourcentage des morceaux dans la carcasse avec la classe de la carcasse. En outre ces auteurs montrent que le pourcentage d'épaule tend à crôitre lorsque la classe de carcasse diminue, alors que le filet et les côtes tendent à avoir plus d'importance dans les carcasses les mieux classées. Il faut remarquer que dans cette expérience, le classement des carcasses en grade est en fait un classement selon le poids, et par suite, les variations constatées ont pour cause principale l'évolution 
relative du poids des morceaux en fonction du poids de la carcasse (Boccard, Dumont et I,EFEBvrE, I 96 I).

Des variations peuvent aussi se produire sur les morceaux de détail. En eflet les carcasses les plus lourdes, c'est-ì-dire les plus grasses, présentent le pourcentage le plus faible pour les meilleurs morceaux (après élimination du gras superflu) (CARpenTer et al., ig64; Fox et al. I964).

A l'appui de la theorie de l'harmonie anatomicue, les travaux de Boccard, Dumont et Peyron (1958) et Boccari) et al., (196I) ont particulièrement bien souligné la constance du pourcentage de gigot à poids de carcasse constant, en calculant des coefficients de corrélation non significativement différents de zéro entre la mesure F (longueur de gigot) et le pourcentage de gigot dans la carcasse sur I 25 et 58 carcasses. "Chez les animux de même poids, la forme du membre postérieur n'entraîne aucune variation significative de la part relative de cette région dans la carcasse."

Ces observations chiffrées éclairent certaines remarfues de IIARING et ScHOMBuRG (I957) et expliquent les faibles variations dans le pourcentage de grigot rapportées par plusieurs auteurs :

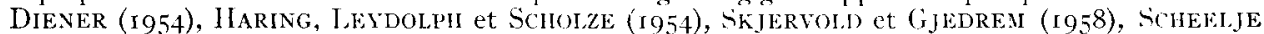
(1959). En outre, Boccarb et Rabomska (1953) montrent que la longueur du gigot (à poids constant) n'a pas de répercussion sur les caractéristiques technologiques de ce morcealu, dans des races de même format, en particulier sur le rendement à la cuisson.

IIINer et ThORNTON (1962) établissent des relations étroites entre 13 mesures de carcasse et le poids des morceaux de plus grande valeur (gigot - - filet - - côtes, gigot + filet - - côtes + épaule). Mais la détermination du poids de ces morceaux à l'aicle d'une équation de régression multiple comprenant l'ensemble de ces mesures, ou la largeur du corps, n'apporte pas une précision très supérieure à la détermination par le poids de carcasse seul. Il est vraisemblable que les mesures utilisées sont très liées au poids de la carcasse.

Ces résultats vont à l'encontre des conceptions traditionnelles associant la bonne conformation des carcasses avec une composition favorable en morceaux et en tissus. Il fant néanmoins constater que ces traditions orientent encore la demande vers des carcasses à gigot court qui bénéficient des prix les plus élevés.

\section{Relation composition tissulaire-qualité de carcasse}

Les résultats du calcul des relations entre les mesures du gras sur la carcasse et les classes de qualité traduisent les variations d'appréciation du gras selon les marchés. Dans le cas des marchés anglo-saxons ces relations sont positives. Les carcasses les mieux notées sont en général plus riches en gras à tous les niveaux (PAuL, Torten et Spuriook, 1964). Le tissu gras peut être mesuré par dissection tissulaire ou par analyse chimique (CLARKE et MCMEFKAN, I9.2 ; HANKINS, I952 ; KEMP, Bull et BEAN, 1953) ou est évalué par la mesure C (épaisseur du gras au-dessus du muscle long dorsal) (Clarke et McMeekan, i952; Stouffer et al., 1958, Mattinew et al., ig60). I.es agneaux de Clarke et McMeekan (1952) représentatifs du marché d'exportation néo-zélandais, et de STouFFER et al. (1958) se classent dans le même ordre pour la qualité des carcasses et pour l'épaisseur C. De même les corrélations entre $\mathrm{C}$ et la note de carcasse sont positives (MatTinews et al. I960), C étant estimée sur animal vif $(r=+0,5$ I et $+0,40$ hautement significatifs) ou mesuré après découpe $(r=+0,36$ significatif et $+0,6 \mathrm{I}$ hautement significatif) (corrélations calculées à poids vif constant).

Cependant CARPEnTER et al. (I964) trouvent des liaisons négatives entre les mesures de gras et la valeur des carcasses (prix de vente par livre de carcasse). Ces méthodes semblent correspondre à une évolution récente du marché américain. Les auteurs concluent que le pourcentage de gras éliminé par parage de la carcasse constitue la meilleur mesure d'estimation de la valeur des carcasses.

En France les carcasses peu grasses sont appréciées, et des études seraient à entreprendre afin de se rendre compte objectivement de l'incidence de l'état de gras sur le prix des carcasses

\section{Relation qualité de la viande-qualité des carcasses}

STOUffer et al. (1958) ont recherché si les carcasses les mieux classées se différenciaient également pour la qualité de leur viande. Ils montrent que le classement est le même pour la qualité commerciale et la note de marbrure de la section du longissimus dorsi avec une corrélation assez élevée $(r=+0,7$ I 5$)$.

Il semble par ailleurs que les notes de marbrure soient d'autant plus fortes que le pourcentage de gras dans les carcasses est plus élevé. (Paul, TorTen et Spurlock, ig64). 
La relation tendreté, classe de carcasse est moins nette (dureté mesurée à l'appareil de Warner-Bratzler) (STOUFFER et al., 1958). (tableau 6)

Remarquons cependant que les carcasses les mieux classées ne possèdent pas la viande la plus tendre. Il est difficile toutefois de tirer des conclusions de grande portée de travaux rares et isolés.

\section{TABLEAU 6}

Liaison entre qualité commerciale, note de marbrure et indice de dureté (d'après STOUFFER et al, I958)

\begin{tabular}{l|c|c}
\hline Qualité commerciale & Indice de dureté & Note de marbrure \\
& & \\
\hline Prime $\ldots \ldots \ldots \ldots \ldots \ldots$ & 8,5 & 10,5 \\
Chock $\ldots \ldots \ldots \ldots \ldots \ldots$ & 7,6 & 9,4 \\
Good $\ldots \ldots \ldots \ldots \ldots \ldots$ & 9,9 & 7,8 \\
Utility $\ldots \ldots \ldots \ldots \ldots \ldots$ & 12,3 & 5,5 \\
\hline
\end{tabular}

\section{V. - CONCLUSION}

Une étude complète de la conséquence actuelle des différents critères (poids, conformation. composition tissulaire, qualité de la viande) sur la qualité des carcasses d'agneaux mesurée soit par une note subjective soit par un prix payé au $\mathrm{kg}$ de carcasse devrait s'efforcer d'apprécier la part revenant à chaque facteur dans la variation de cette qualité. Le modèle mathématique, servant de base à l'étude, pourrait donc comprendre des variables indépendantes : poids, longueur $\mathbf{F}$, mesure C, note de qualité de la viande, et devrait tenir compte aussi des interactions susceptibles d'exister entre ces variables (poids et gras par exemple). Les travaux de cette nature sont rares. On ne doit pas perdre de vue également que le prix au $\mathrm{kg}$ est susceptible de variations qui tiennent à la nature du marché et à la saison (milieu économique) (Phillips, Card et Carter, ig6o) et dont il faudrait aussi pouvoir tenir compte.

Les études de la composition des carcasses mettent en relief l'originalité du tissu gras par rapport aux tissus musculaires et osseux : cette originalité, fonctionnelle au niveau de l'animal vivant, puisque le gras ne semble remplir aucun rôle moteur ou structural, se traduit surtout par des vitesses de dépôt différentes. Tout accroissement de poids d'un animal est dû à une part de plus en plus grande d'accroissement du tissu gras au cours de son développement, alors que les pourcentages relatifs de muscle et d'os restent beaucoup plus proches les uns des autres. Il serait intéressant d'étudier les variations du rapport os/muscle, selon le poids de la carcasse et selon la race, les variations du gras étant exclues.

Il en résulte que sur un grand nombre de carcasses les seuls éléments vraiment variables sont le poids, l'état d'engraissement et la conformation. Le poids détermine la taille des morceaux mais moins sensiblement leur part relative. Par ailleurs l'état d'engraissement d'une carcasse n'est pas indépendant de son poids : pour une race donnée les carcasses les plus lourdes sont aussi les plus grasses (JUDGE et al. (1963), trouvent $r=+0,70$ entre le pourcentage d'excès de gras et le poids de la carcasse).

S'il s'agit de déterminer la race qui conduira au type idéal du marché, il faut choisir celle qui donnera les carcasses idéales en poids et en gras avec la croissance la plus rapide. C'est ce qui peut expliquer l'intérêt actuel porté aux agneaux Ile-de-France ou aux Berrichons du Cher. L'aspect conformation ne présentant plus que l'intérêt d'une coquetterie ou d'une mode, les éleveurs peuvent être incités à délaisser la recherche de gigots courts. Déjà dans les conditions actuelles de nombreux marchés, le profit maximum est obtenu en recherchant d'abord le maximum de prolificité afin d'obtenir le maximum d'agneaux sevrés dont le poids commercial est atteint dans les meilleurs délais (taux de fécondité et de croissance élevés) (WINTERs, I 944, cité par HazEL et Terill, I946. Bywater, I945; Walker, I949; Coleman et McDonald, I952 ; Coleman et Godlee, I952 ; Scholze, I954 ; COOP, I957; BRADFord, Weir et TORELl, i960). 
Il semble donc que les travaux de recherche doivent orienter leurs efforts vers la détermination de la composition des carcasses et la variation des différents composants tissulaires, et sur le contrôle de races joignant un taux de fécondité élevé à une vitesse de croissance optimum dans les conditions fourragères locales.

Reçu pour publication en jantier 1966.

\section{SUMMARY}

QUALITY RATING OF LAMB CARCASSES

I. - Introvuction.

II. - Quality criteria of a carcass.

$I^{\circ}$ Carcass weight

$2^{\circ}$ Carcass conformation

$3^{\circ}$ Carcass composition

$4^{\circ}$ Quality of meat

III. - Estimation of cakcass characteristics.

$I^{\circ}$ Direct methods
a) Carcass weight
b) Carcass conformation
c) Carcass composition
d) Quality of meat

$2^{\circ}$ Indirect methods

a) Indirect estimation of carcass weight

b) Estimation of carcass conformation on live lamb

c) Indirect estimations of carcass composition

- Estimation by dressing yield

- Estimation by carcass weight

- Estimation by specific gravity

- Estimation by potassium-40

- Estimation by various linear measurements

- Estimation by composition of sample cuts

IV. IJISCUSSION.

$1^{\circ}$ Relationship between weight and carcass quality

$2^{\mathrm{O}}$ Relationship between conformation and carcass quality

$3^{\mathrm{o}}$ Relationship between proportion of cuts and carcass quality

$4^{\text {o }}$ Relationship between composition of tissues and carcass quality

$5^{\circ}$ Relationship between quality of meat and quality of carcass

V. - Conclusion.

\section{RÉFÉRENCES BIBLIOGRAPHIQUES}

Ament D. L.., (ialgan M. W., Rupnow I. II., I962. Carcass characteristics of Columbia market lambs (Abstr.) J. anim. Sci., 21, 977-978.

Associntion of Official Agricultural Chemists, 1960. Official methods of analysis. 9th ed. $297-304$. Association of Official Agricultural Chemists, Washington, D. C. $832 \mathrm{p}$.

DE BACA R. C., BOGART R., 1959. Factors affecting condition and conformation of crossbred spring lambs. J. anim. Sci., 18, 1094-1 102.

Bailey C. M., Pope A. L., Cimapman A. B., 1961. Predicting loin-eye area in lambs from weaning traits and carcass measurements. J.anim. Sci., 20, $3^{\circ 2-306 .}$ 
Barton R. A., Phillips 'T. O., Clarke E. A., 1949. Influence of sire on fat lamb quality. Proc. N.Z. Soc. Anim. Prod., 9, 66-84.

BARTON R. A., KIRTON A. H., 1956. Determination of fat in mutton carcasses by measurements of specific gravity. Nalure, 178, 920.

Barton R. A., KirTon A. II., ig58 a. Carcass weight as an index of carcass components with particular reference to fat. J. Agric. Sci, 50, $333^{1-334}$.

BARTON R. A., KIRTON A. H., 1958 b. The leg and the loin as indices of the composition of New Zealand lamb and mutton rarcasies. N. Z. J. Agric. Res., 1, 78.3-789.

Bartox R. A., KIRTON A. H., 1958. Assessment of fat in mutton and lamb. Proc. N. Z. Soc. Anim. Prod., 18, I 12 -I 25.

Boccarb R., Dunoxt B. I., ro55. Etude de la production de la viande chez les Ovins. I. Ia coupe des carcasses. Définition d'unc découpe de référence. 1 mn. Zootech, 3,241 .

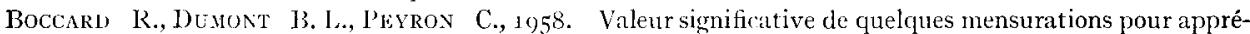
cier la qualité des rarcasse's d'agneaux. 4h heet. Lurop. meat research workers Cambridge., Sept. I 5-19th.

Boccarv) R., Dumont B. L., i 960 a. Etude de la production de la viande chez les Ovins. II. Variation de l'importance relative des clifférentes régions corporelles de l'agneau de boucherie. Ann. Zootech., 9, $355^{\sim} 3^{6} 3$.

Boccals) R., Jumont 13. L., I gro b. Note sur la mesure et la variation de l'adiposite des carcasses d'agneau. VIe Réunion des Insiliuts le Recherche sur les Viandes., Ut recht 20 août, 3 septembre.

Boccaru R., Dunont 13. L., Le Guelte J., Arnoux J., ighr. Étude de la production de la viande chez les Ovins. IV. Relation entre la forme et la composition du membre postérieur. Ann. Zootech., 10, I $55^{-160 .}$

Boccard R., Dumont B. L., Lisitibvre J., 1962. Étude de la production de la viande chez les ovins. V. Note sur la croissance relitive des régrions corporelles de l'agneau. Ann. Zoolech., 11, $257-262$.

Boccari, R., Radonska II. J., 1963. Étude de lat production de la viande chez les Ovins. VI. Influence de la forme du membre postéricur sur ses caractéristiques technologiques. Ann. Zoolech., 12, 5-15.

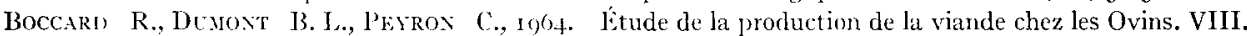
Relations entre les dimensions de la carcasse d'agneau. Amm. Zootech., 13, 307-378.

Botkin M. P'., Marion S'TAnete, Schoonover C. (9., 195\%. Relationship between live lamb measurements and meatiness. (West. Sect. Mbstr.). J. anim. Sci., 18, I 165.

BraDioris G. E., Whir W. C., TokliLl D. T., igfo. Growth rate, carcass grade, and net returns of Suffolk and Southdoa'n -sired lambs under ranger conditions. J. anim. Sci., 19, 493-501.

BRAY R. W., 1963. Symposium on feed and meats terminology. IV. Quantitative measures of carcass composition and qualitative cvaluations. J. anim. Sci., 22, 548-554.

Brier G. W. SCHOTT R. (S., simmons W. L., I9to. The discriminant function applied to quality rating in shcep. Proc. Aner. Soc. Anim. Prod., 1940, 153-160.

Bywater T. L., 1945. Crossbrceding of sheep. J. R. Agric. Soc. Ingl., 106, 166-184.

Campbele D., Stonaker H. H., Espitin A. L., 1959. The use of ultrasonics to estimate the size of the longissimus dorsi muscle in sheep. (Abstr.). J. anim. Sci., 18, r483.

Carpenter Z. L., King G. T., Orts F. A., Cunninghan N. L., I964. liactors influencing retail carcass value of lambs. J. anim. Sci., 23, 74I-745.

Carroli M. A., O'Carrol. F. M., ig6. Differences between left and right sides of lamb carcasses. Irish. J. Agric. Res.2 3, $223^{-2} 3^{8}$.

Clarke E. A., Mcileekax C. P., 1952. New Zealand lamb and mutton. N. Z. J. Sci. Techn., A 33 I-I 5 .

Coleman J. M., r95I. Fat lamb production : iuheritance of tleshing qualities. Agric. Gaz. N. S. W., 62, $58-60$.

Coleman J. M., (aobles A. C., 1952. Early lamb production on irrigated pastures. Agric. Gaz. N.S.W., 63, 228.

Coleman J. M., McDonalt, i952. Fat lamb raising on irrigation areas. Results of breeding and supplementary feeding trials. Agric. Gaz. N.S.W., 63, 345-350.

Coop I. E., 195\%. Rorder Leicesier cross ewes for fat lamb production. Proc. N. Z. Soc. Anim. Prod., 17, $88-95$.

Coway R. L., Keck E. J., Ziegler J. H., Watkins J. L., Miller R. C., ig6i. Relationship of specific sravity to fat and energy content of lamb carcasses. (Abstr.). J. anim. Sci., 20, 916.

Davis D. L., Ockermai H. W., Cahlll V. R., Tyznik W. J., Parker C. F., ig64. Ultrasonic evaluation of lambs. J. anim. Sci., 23, I202.

Delage J., l'oly J., Vissac B., I955. Étude de l'efficacité relative de diverses formules de barymétrie applicables aux bovins. Ann. Zoolech., 4, 219-23r.

Diener H. O., I954. Fleischproduktion und Fleischquatität der Merinos-landschafrasse. Tierzüchter, 8, 2 I $7-220$.

Dinkel C. A., Wilson T. L., Tuma H. J., Minyard J. A., ig65. Ratios and percents as measures of carcass traits. J. anim. Sci., 24, 425-529. 
Dumont B. L., r955. L'utilisation de l'antipyrine pour la mesure in vivo de l'eau totale du corps chez les ovins. Ann. Z̈ootech., 4, 315-319.

ElsLey F.W. H., MCDOyali) I.. Folyler V. R., 1964. The effect of plane of nutrition on the carcasses of pigs and lambs when variations in fat content are excluded. Anim. Prod., 6, I4I-I54.

Esipin A. I., Henry L. G., Sutherlani 'T. M., 1064. The regression method for adjusting loin eye area for differences in lamb carcass weights. (Abstr.). J. anim. Sci., 23, 859.

F. H. Z. (Fédération EURol'́enNe de ZoOTHCHNIE), 1953. Production et commercialisation de la viande. V. Jues principales découpes europécmnes. C. R. 3imes jonrnées d'élude de la F.E.Z., Sienne-Rome, I 953 , 137 -I 46 .

Field R. A., Kemp J. D., Varney W. Y., 1963. Indices for lamb carcass composition. J. anim. Sci., 22, $218-221$.

Fox C. W., Kennick W. II, Eller R., II Adrthur J. A. B., 1964. Ram evaluation based on retail cut-out value of their progeny. I. anim. Sci.. 23, 859 .

Garret W. N., Meyer J. II., Lofgrees G. P., 1959. The comparative energy requirements of sheep and cattle for maintenance and gain. J. anim. Sci., 18, 528-547.

HAmMond J., 1932. Growth and development of mullon qualilies in the sheep. Oliver and Boyd. Ed. London.

Hammond J., 1936. The shape of the longissimus dorsi muscle in domestic animals. N. Forsh. Tierz. Anstam. (cité par BoCCARD, Dumont et PEYRon, 1058).

HaNkINS O. G., kLIIS N. R., I93\%. Fat in relation to quantity and quality factors of meat animal carcasses. Proc. Amer. Soc. Anim. Prod., 32th, 314-310.

Hankins O. G., I947. Estimation of the composition of lamb carcasses and cuts. U.S. Dep.Agric. Washington Tech. Bull., 944 .

Hankins O. G., Hiner R. I., Simmons V. I., I951. A study of meat characteristics of Karakul sheep. J. anim. Sci., 10, 399-410.

Haring F., Leydolpil W., Sciolze F., 1954. Lämmenmastversuch mit verschiedenen Schafrassen und Kreuzungen, zugleich eine Frage zur Methodik Schafmastleistungs prüfung. Ziüchtingskunde, 26, $25^{-33}$.

Haring F., Schomburg K., i 957. Milchlämmermast mit Lümmern verschiedener Schafrassen. Tierziuchter, 1,2 .

Hazrel L. N., Terrill C. E., I946. Heritability of type and condition in range Rambouillet lambs as evaluated by scoring. J. anim. Sci., 5, 55-61.

Hillman M., Whent J. D., Menzies C., Mackintosi D. L., Merkel R. A., ig6z. Heritabilities of and correlations between carcass and live animal traits in sheep. (Abstr.). J. anim. Sci., 21, 973.

Hiner R. L., Thornion J. W., I962. Study of certain lamb and carcass quality factors. J. anim. Sci., 21, 5 I I-5 I5.

HIRzEL R., 1939. Factors affecting quality in mutton and beef with special reference to the proportion of muscle fat and bone. Onder. J. Vet. Sci. Anim. Ind., 12, 379-550. (Cité par Boccard, Dunont et I'EYRON, $\left.195^{8}\right)$.

Hundt K. W., 1959. Körperformen, Fleichleistung und Schlachtgualität bei Mastlämmern. Zïchtungskunde, 31, I 58-1 70 .

Huxley J., 1932. Problems of Relalive groioth. Wethuen. London.

Judge M. D., Martin T. (;., 1963. Prediction of lamb carcass composition from carcass weights and measurements (Abstr.). J. anim. Sci., 22, 828.

Judge M. D., Stor M., Kessler W. V., Cirristian J. J., 1963. Lamb carcass and live lamb evaluations by potassium-40 and carcass measurements. J. anim. Sci., 22, 418-421.

KatAdA A., TAKEDA I., I958. The relationship of body type to economic characters of corriedale fat wether lamb in Japan (en japonais). Jap. J. Zoolech. Sci., 29, 49-53 (in Anim. Breed. Abstr., 2\%, 260).

Kaukfman R. G., Norton H. W., ig64. Distribution of potassium in muscle tissue (Abstr.). J. anim. Sci., 23, I203.

KemP D. J., Bull S., BeAN H. W., 1953. The economy and nutritive value of different cuts of lamb of different grades. J. anim. Sci., 12, 338-346.

KEMP J. D., BARTON R. A., I965. A comparison between two methods of chemically analysing lamb cuts and carcasses, and a proposal for estimating ash from regression equations. $\Lambda . Z$. J. Agric. Res., 8, $58-62$.

Khandfikar V. N., McManus W. R., Goldstone C. L., 1963. Measurements of length of the forecannon bone in live sheep. Nature 197, 92-93.

Kirton A. II., I964. Assessment of body composition in the live animal. Proc. N. Z. Soc. Anim. Prod., 24, $77-89$.

Kirton A. I1., Barton R. A., 1958. Specific gravity as an index of the fat content of mutton carcasses and various joints. N. Z. J. Agric. Res., 1, 633-641.

Kirton A. H., BARTon R. A., ig62. Study of some indices of the chemical composition of lamb carcasses. J. anim. Sci., 21, 553-557.

Krrton A. H., Barton R. A., Rat A. L., ig62. The efficiency of determining the chemical composition of lamb carcasses. J. agric. Sci., 58, $3^{81-386 .}$

Kirton A. H., Pearson A.M., Nelson R. H., Anderson E. C., Schuch R. L., ig6i. Use of naturally occurring potassium 40 to determine the carcass composition of live sheep. J. anim. Sci., 20, 635-639. 
Kirton A. H., Pearson A. M., i 963 . Comparison of methods of measuring potassium in pork and lamb and prediction of their composition from sodium and potassium. J. anim. Sci., 22, 125-131.

Kirton A. H., UlyatT M. J., Barton R. A., I959. Composition of some Fat-free carcasses and bodies of New Zealand sheep. Nalure, 184, Suppl. 22, 1724.

KnigitT A. D., Foote W. C, Bennett J. A., I959. Some relationships of live animal and carcass measurenents to carcass characteristic's and breed type differences in lamb. (Abstr.). J. anim. Sci., 18, i I67.

Large R. V., Tayler J. C., 1954. Studies on the growth of Clun lambs. Emp. J. Exp. Agric., 22, I4I-147.

Lathay S. D., Moody U., KenP J. D., WoOlfolk P. (i., I964. Reliability of predicting lamb carcass composition (Abstr.). J. anim. Sci., 23, 861.

MCMELKan C. P., igoo. Growth and development in the pig, with special reference to carcass quality characters. J. Igric. Sci., 30, 276 .

Matthews D. J., Merkel R. A., Whent J. D., Cox R. F., igoo. A technique for measuring the crosssectionnal areas of the longissimus dorsi nuscle and fat depth in lambs. J. anim. Sci., 19, 803-809.

Milford R., Minson D. J., I 965 . The energy value of ryegrass and cocksfoot assessed by a slaughter technique with lambs. Brit. J. Nitr., 19, 373-382.

Munson A. W., WiItpman J. V., Walters L. F., I965. A method for estimating ether extract in lamb carcasses (Abstr.). J. anim. Sci., 24, 282.

Neville W. E. jr, Chapmax A. B., POpF A. L., 1958. Comparison of lambs from Western (ColumbiaRambouillet) ewes and sired by rams of four Doton breeds. J. anim. Sci., 17, 763-773.

Orme L. F., Cintstian R. F., Beil. T. D., ig63. Iive animal and carcass indices for estimating the carcass composition in lambs. J. anim. Sci., 21, 666.

PALsson H., 1939-1940. Meat qualities in the sheep, with special references to Scottish breeds and crosses. J. Agric. Sci., 29, $560,30.1$.

Palsson H., Verges J. 13., 1950. Effects of the plane of nutrition on growth and the development of carcass quality in lambs. I. The effects of high and low planes of nutrition at differents ages. J.Agric. Sci., 42, I-92.

Palsson H., Vkrges B. J., I950. Effects of the plane of nutrition on growth and the development of carcasse quality in lambs. II. Effects on lambs of $30 \mathrm{lb}$ carcass weight. J. Agric. Sci., 42, 93-139.

Paul P. C., Tortex J., spurlock G. M., I 964 . Wating quality of lamb. I. Effect of age. II. Effect of preslaughter nutrition. III. Over-all comparisons and interrelationships. Food Technol., 18, 12 I-I3O.

Phillips C. D., Cari) D. C., Carter R. L., I960. (haracteristics of prices paid for Kentucky spring lambs, Agric. Exp. Stal. Univ. Kentucky, 670.

Robinson J. J., Brive F. li., Dor; A. C.., i956. Fat lamb studies in Victoria. I. An assessment of the relative value of various external measurements for differenciating beetween various grades of export lamb carcasses. Aust. J. Agric. Res., 7, 345-365.

Rovvier R., RICARI) F. II., I965. Étude des mesures de conformation du poulet. II. Recherches des composantes de la variabilité morphologique du poulet vivant. Ann. Zoolech., 14, 213-227.

Rouvier R., VisSAC B., 1964. Application des méthodes d'analyse factorielle à l'étude de la variabilité morphologique de carcasses de bovins adultes. Biométrie-Praximétrie, 5, 93-11 I.

Scheelje R., 1959. Auswertungen der Nachkommen : Schaftsprüfungen auf Mastleisst ung und Schlacht körperqualitat an Böcken des Merinosfleischajes in einer Testherde. Zitchlungskunde, 31, 366-377.

Scheper J., Ig62. Uber Merkmale der Fleischbeschaffenheit und die Möglichkeit ihrer Beeinflussung von der Schlachtung. Z. Tierz. Ziicht Biol., 7\%, $420-+60$.

Scholze F., 1954. Die Junglammemast beim Leineschaf under besonderer berück sichtigung von Milchleistung und futterverwertung. Z. Tiers. Zïchl Biol., 64, 25-46.

Shorland F. B., DF la Mare P. B. D., Sorrel D. M. P., Barnicoat C. R., 1947. Simplificd procedures for determining the nutritive value of carcasses with special reference to New Zealand lamb and mutton. N. Z. J. Sci. Tech., 29, 76-98.

SKJervold H., GJEIREM T., 1958. Kjo ttproduksjonstgenskapene hos tre saueraser og to krysningsgrupper. Meldinger fra Norges Landbrukshogskole, $3 \%$, 1 .

Starke J. S., Joubert D. M., i96r. A score card for lamb and mutton carcasses. J. Agric. Sci., 57, 3 I $9-323$.

Stouffer J. R., Hogue D. E., Marden D. H., Weilington G. H., I958. Some relationships between live animal measurements and carcass characteristics of lamb (Abstr.). J.anim. Sci., 17, II5I.

SuCK II. J., 1957. Untersuchungen an deutschen schwarckopfigent Fleischschalen hinsichllich des Einflusses von Original-Hampshire Down. Bócken auf Wolleishung und Körperentwicklung in zwei kurhessischen Stamm herden. Institut für Tierzucht und Vilchwintschaft Justus Liebig. Universitht. Griessen.

TANEJA G. C., $1955(a)$. The relative importance of heredity and enviromment in body weight increments at different ages in Australian Merino sheep. Aust. J. Igric. Res., 6, 347-349.

Taneja G. C., I955 (b). Mution qualities in Australian Merino sheep. Aust. J. Agric. Res., 6, 882-890.

TANner J. ll., BurT A. W. A., 1954. Ihysique in the infra human mammalia: A factor analysis of body measurements of dairy cows. $J$. Genel., 52, 36-51.

TAylor St. C. S., Rollns W. C., ro63. Body size and conformation in identical twin cattle. Anim. Prod., 5, 77-86. 
Thwaites C. J., Yeates N. T. M., Pogue R. F., ig64. Objective appraisal of intact lamb and mutton carcasses. J. Agric. Sci., 63, 415-420.

Timon V. M., I963. Indices of lamb carcass composition. (Proc.). Anim. Prod., 5, 219.

Tulloh N. M., I963. Relation between carcass composition and live weight of sheep. Nature, 197, 809-8 ro.

Ulyatt M. J., Barton R. A., I963. A comparison of the chemical and dissectible carcass composition of New Zealand Rommey Marsh ewes. J. Agric. Sci., 60, 285-289.

Walker D. E., McMeekan C. P., I944. Canterbury lamb N. Z. J. Sci. Tech., (A), 26, 51-I20, cités par Boccard, Dumont et Peyron (i958).

Malker D. E., I949. North Island fat lamb crosses (Summary). Proc. N. Z. Soc. Anim. Prod., 9, Io7-III.

WALLACE L. R., I948. The growth of lambs before and after birth in relation to the level of nutrition. J. Agric. Sci, 38, 93-153, 243-302.

Winters L. M., i944. Cité par Hazel et Terril. (I946, J. Anim. Sci., 5, 55-6I) An analysis of the factors affecting productive value in sheep breding. (Abstr.). J. anim. Sci, 3, 435 .

WRIGHT S., I932. General group and special size factors. Genetics, 17, 604-6 5 .

Wright S., I954. In : Kempthorne O., Bancroft T. A., Gowen J. W., Lush J. L., Statislics and mathematics in biology, II-33. The Iowa State College Press. Ames, Iowa.

Zobrisky S. E., Moody W. G., Ross C. V., Naumann H. D., Hebrick H. B., 196r. Live animal and carcasse indices of lamb composition (Abstr.). J. anim. Sci., 20, 922. 